\title{
Additive conjoint measurement with ordered categories ${ }^{1}$
}

\author{
Denis Bouyssou ${ }^{2}$ \\ CNRS - LAMSADE \& \\ Université Paris Dauphine \\ Thierry Marchant ${ }^{3}$ \\ Ghent University
}

First version: 12 May 2008

First revised version: 2 March 2009

Second revised version: 14 July 2009

Typos corrected: 17 August 2009 \& 26 January 2010

\footnotetext{
${ }^{1}$ Authors are listed alphabetically. During the preparation of this paper, Denis Bouyssou was supported by the Belgian Fonds National de la Recherche Scientifique and Thierry Marchant benefited from a visiting position at the Université Paris Dauphine. This support is gratefully acknowledged. We would like to thank David H. Krantz for his help with one of the examples in Krantz et al. (1971, Ch. 6) and his suggestion in Krantz (2009) of the principle used in Example A-5 in the supplementary material to this paper.

${ }^{2}$ CNRS, LAMSADE, Université Paris Dauphine, Place du Maréchal de Lattre de Tassigny, F-75 775 Paris Cedex 16, France, tel: +33 1440548 98, fax: +331440540 91, e-mail: bouyssou@lamsade.dauphine.fr. Corresponding author.

${ }^{3}$ Ghent University, Department of Data Analysis, H. Dunantlaan, 1, B-9000 Gent, Belgium, tel: +32 926463 73, fax: +32926464 87, e-mail: thierry.marchant@UGent. be.
} 


\begin{abstract}
Conjoint measurement studies binary relations defined on product sets and investigates the existence and uniqueness of, usually additive, numerical representations of such relations. It has proved to be quite a powerful tool to analyze and compare MCDM techniques designed to build a preference relation between multiattributed alternatives and has been an inspiring guide to many assessment protocols. The aim of this paper is to show that additive representations can be obtained on the basis of much poorer information than a preference relation. We will suppose here that the decision maker only specifies for each object if he/she finds it "attractive" (better than the status quo), "unattractive" (worse than the status quo) or "neutral" (equivalent to the status quo). We show how to build an additive representation, with tight uniqueness properties, using such an ordered partition of the set of objects. On a theoretical level, this paper shows that classical results of additive conjoint measurement can be extended to cover the case of ordered partitions and wishes to be a contribution to the growing literature on the foundations of sorting techniques in MCDM. On a more practical level, our results suggest an assessment strategy of an additive model on the basis of an ordered partition.
\end{abstract}

Keywords: Additive conjoint measurement, Multiple criteria analysis, Sorting, Ordered categories. 


\section{Contents}

1 Introduction and motivation $\quad 1$

2 Definitions and notation 3

2.1 The setting . . . . . . . . . . . . . . . . 3

2.2 Primitives . . . . . . . . . . . . . . . . . . . . 3

2.3 The model . . . . . . . . . . . . . . . . . 4

3 Intuition of the axioms and sketch of an assessment protocol 4

3.1 Ordering the elements of $X_{i}$ and compatibility . . . . . . . 5

3.2 Strict compatibility . . . . . . . . . . . . . 5

3.3 Uniqueness . . . . . . . . . . . . . . . . . 6

3.4 Conventions and normalization ............. 6

3.5 Building diagonal standard sequences . . . . . . . . . . . . . . . . 7

3.6 The Thomsen condition . . . . . . . . . . . . . . . 8

3.7 Checking and completing the construction . . . . . . . . . . . 11

3.8 At least four attributes . . . . . . . . . . . . . . . . . 12

4 Axioms and Results 13

4.1 Necessary axioms . . . . . . . . . . . . . . . . . . . . 13

4.2 Structural axioms . . . . . . . . . . . . . . . . . . . . . 18

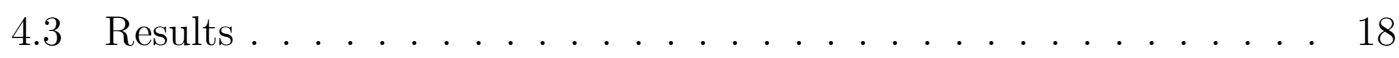

5 Beyond threefold partitions $\quad \mathbf{2 0}$

5.1 The setting . . . . . . . . . . . . . . . . . 20

5.2 The model . . . . . . . . . . . . . . . . . . . 20

5.3 Axioms and result . . . . . . . . . . . . . . . . 21

6 Relation to the literature and discussion $\quad 23$

6.1 Relation to the literature . . . . . . . . . . . . . . . . 23

6.2 Discussion . . . . . . . . . . . . . . . . 25

$\begin{array}{ll}\text { Supplementary material } & 27\end{array}$

$\begin{array}{ll}\text { A Proof of Proposition } 1 & 27\end{array}$

A.1 Preliminary results . . . . . . . . . . . . . . . . . . 27

A.2 Proof of Proposition 1: existence . . . . . . . . . . . . . . 30

A.3 Proof of Proposition 1: uniqueness . . . . . . . . . . . . . . . . 31 
B Refining conditions $\quad 32$

B.1 Proof of Theorem 1 . . . . . . . . . . . . . . . . . . 32

B.2 Proof of Theorem $2 \ldots \ldots \ldots \ldots$

B.3 Examples . . . . . . . . . . . . . . . . . . 35

$\begin{array}{ll}\text { C Proofs with more than two categories } & 39\end{array}$

C.1 Proof of Theorem 3: existence . . . . . . . . . . . . . . . . . . . 39

C.2 Proof of Theorem 3: uniqueness . . . . . . . . . . . . . . . . 42

$\begin{array}{ll}\text { References } & 42\end{array}$ 


\section{Introduction and motivation}

The aim of most MCDM techniques (see Belton and Stewart, 2001, Bouyssou et al., 2006, for recent reviews) is to build a model allowing to compare alternatives evaluated on several attributes in terms of preference. Conjoint measurement (see Krantz et al., 1971, Ch. 6 \& 7 or Fishburn, 1970, Ch. 4 \& 5) is a branch of measurement theory studying binary relations defined on product sets and investigating the existence and uniqueness of, usually additive, numerical representations of such relations. Denoting by $\succsim$ an "at least as good as" preference relation on the set of alternatives, the central model in conjoint measurement is the additive value function model in which,

$$
x \succsim y \Leftrightarrow \sum_{i=1}^{n} v_{i}\left(x_{i}\right) \geq \sum_{i=1}^{n} v_{i}\left(y_{i}\right),
$$

where it is understood that $x$ and $y$ are alternatives and that $x_{i}$ denotes the evaluation of alternative $x$ on the $i$ th attribute. The real-valued functions $v_{i}$ numerically recode the evaluations of the alternatives on the various attributes, in such a way that alternatives can simply be compared taking the sum of these functions.

Additive conjoint measurement has proved to be a powerful tool to analyze and compare MCDM techniques. It has also been an inspiring guide to many assessment protocols, i.e., structured way to interact with a decision maker (DM) in order to build an additive numerical representation and, therefore, to structure his/her preferences (see, e.g., Keeney and Raiffa, 1976 or von Winterfeldt and Edwards, 1986).

In some instances, building a recommendation on the basis of a preference relation between alternatives does not seem to be fully adequate. Indeed, a preference relation between alternatives is an evaluation model that has only a relative character. Hence, it may well happen that the best alternatives are not desirable at all. This calls for MCDM techniques building evaluation models having a more absolute character. Such models belong to what Roy (1996) called the "sorting" problem statement. Suppose, e.g., that an academic institution wants a model that would help the committee responsible for the admission of students in a given program. A model only aiming at building a relation comparing students in terms of "performance" is unlikely to be much useful. We expect such an institution to be primarily interested in a model that would isolate, within the set of all candidates, the applicants that are most likely to meet its standards defining what a "good" student is.

MCDM techniques designed to cope with such problems most often lead to build a partition of the set of alternatives into ordered categories, e.g., through the comparison of alternatives to "norms" or the analysis of assignment examples. This type of techniques has recently attracted much attention in the literature (see 
Greco et al., 1999, 2002a,b, Zopounidis and Doumpos, 2000a, 2002, for reviews). For instance, the UTADIS technique (see Jacquet-Lagrèze, 1995, Zopounidis and Doumpos, 2000b) uses an additive value function to sort alternatives between several ordered categories.

The aim of this paper is to contribute to a recent trend of research (see Bouyssou and Marchant, 2007a,b, Słowiński et al., 2002) aiming at providing sound theoretical foundations to such methods. Słowiński et al. (2002), extending Goldstein (1991), have concentrated on models admitting a "decomposable" numerical representation that have a simple interpretation in terms of "decision rules" and investigated some of its variants. Bouyssou and Marchant (2007a,b) have studied a model that is close to the one used in the ELECTRE TRI technique (see Mousseau et al., 2000, Roy and Bouyssou, 1993) that turns out to be a particular case of the decomposable models studied in Słowiński et al. (2002).

This paper concentrates on additive numerical representations. We will suppose that alternatives evaluated on several attributes are presented to a DM. These attributes describe features of the alternatives that are pertinent to the DM and use various types of scales. Instead of comparing alternatives by pairs in order to define a preference relation, the DM is expected to answer by specifying for each alternative if he/she finds it "attractive" (better than the status quo), "unattractive" (worse than the status quo) or "neutral" (equivalent to the status quo). We show how to build an additive representation, with tight uniqueness properties, using such an ordered partition of the set of objects. Our main objective will be to show how to adapt the classical results of conjoint measurement characterizing the additive value function model (i.e., the ones presented in Krantz et al., 1971, Ch. $6 \& 7$ ) to the case of ordered categories (a related path was followed by Nakamura, 2004, for the case of decision making under risk). In performing such an adaptation, we will try to keep things as simple as possible. A companion paper (Bouyssou and Marchant, 2009) is devoted to the more technical issues involved by such an adaptation.

The rest of this paper is organized as follows. We introduce our setting in Section 2. Section 3 informally discusses our axioms and gives the sketch of an assessment protocol for our model. Section 4 contains our main results. Section 5 shows how to extend these results to cover more general cases involving several ordered categories. A final section discusses our findings and, in particular, relates them to Vind's (1991). For space reasons and with apologies to the reader, most proofs are relegated to the supplementary material to this paper. 


\section{Definitions and notation}

\subsection{The setting}

Let $n \geq 2$ be an integer and $X=X_{1} \times X_{2} \times \cdots \times X_{n}$. The interpretation is as follows. We have alternatives evaluated on a set $N=\{1,2, \ldots, n\}$ of attributes. The set $X_{i}$ gathers all possible levels that an alternative can possibly take on the $i$ th attribute. The set $X$ is the set of all possible vectors of evaluations on the $n$ attributes. As is customary in conjoint measurement, we identify the set of all alternatives with $X$. Therefore, elements $x, y, z, \ldots$ of $X$ are interpreted as alternatives evaluated on a set of $n$ attributes.

For any nonempty subset $J$ of the set of attributes $N$, we denote by $X_{J}$ (resp. $\left.X_{-J}\right)$ the set $\prod_{i \in J} X_{i}\left(\operatorname{resp} . \prod_{i \notin J} X_{i}\right)$. With customary abuse of notation, when $x, y \in X,\left(x_{J}, y_{-J}\right)$ will denote the element $w \in X$ such that $w_{i}=x_{i}$ if $i \in J$ and $w_{i}=y_{i}$ otherwise. We often omit braces around sets and write, e.g., $X_{-i}, X_{-i j}$ or $\left(x_{i}, x_{j}, y_{-i j}\right)$. This should cause no confusion.

\section{$2.2 \quad$ Primitives}

The traditional primitive of conjoint measurement is a binary relation $\succsim$ defined on $X$ with $x \succsim y$ interpreted as " $x$ is at least as good as $y$ ". Our primitives will consist here in the assignment of each object $x$ to a category that has an interpretation in terms of the intrinsic desirability of $x$.

More precisely, we suppose that alternatives in $X$ are presented to a DM. For each of these alternatives, he/she will specify whether he/she finds it "attractive" (better than the status quo), "unattractive" (worse than the status quo) or "neutral" (equivalent to the status quo). Similar primitives are considered in Nakamura (2004) for the case of decision making under risk and in Goldstein (1991) in a multiattribute setting similar as ours. This process defines a threefold ordered partition $\langle\mathscr{A}, \mathscr{F}, \mathscr{U}\rangle$ of the set $X$ (note that we abuse terminology here since we do not require each of $\mathscr{A}, \mathscr{F}$ and $\mathscr{U}$ to be nonempty; this will result from the structural conditions used below). Alternatives in $\mathscr{A}$ are judged Attractive. Alternatives in $\mathscr{U}$ are judged Unattractive. Alternatives in $\mathscr{F}$ are judged neutral and therefore lies at the Frontier between attractive and unattractive alternatives. We often write $\mathscr{A} \mathscr{F}$ instead of $\mathscr{A} \cup \mathscr{F}$ and $\mathscr{F} \mathscr{U}$ instead of $\mathscr{F} \cup \mathscr{U}$. The three categories in $\langle\mathscr{A}, \mathscr{F}, \mathscr{U}\rangle$ are ordered. All alternatives in $\mathscr{A}$ are preferable to all alternatives in $\mathscr{F}$ and the latter are preferable to all alternatives in $\mathscr{U}$. It is important to notice that alternatives belonging to $\mathscr{A}$ are not "equally" preferable. Some of them may be quite attractive while others may only be slightly better than the status quo. A similar remark holds for $\mathscr{U}$. 


\section{Definition 1}

We say that $\langle\mathscr{A}, \mathscr{F}, \mathscr{U}\rangle$ is non-degenerate if both $\mathscr{A}$ and $\mathscr{U}$ are nonempty.

We say that an attribute $i \in N$ is influent for $\langle\mathscr{A}, \mathscr{F}, \mathscr{U}\rangle$ if there are $x_{i}, y_{i} \in X_{i}$ and $a_{-i} \in X_{-i}$ such that $\left(x_{i}, a_{-i}\right)$ and $\left(y_{i}, a_{-i}\right)$ do not belong to the same category in $\langle\mathscr{A}, \mathscr{F}, \mathscr{U}\rangle$.

\subsection{The model}

In this paper we concentrate on additive representations of $\langle\mathscr{A}, \mathscr{F}, \mathscr{U}\rangle$, i.e., a model in which there are real-valued functions $v_{i}$ on $X_{i}$ such that, for all $x \in X$,

$$
x \in\left\{\begin{array}{c}
\mathscr{A} \\
\mathscr{F} \\
\mathscr{U}
\end{array}\right\} \Leftrightarrow \sum_{i=1}^{n} v_{i}\left(x_{i}\right)\left\{\begin{array}{l}
> \\
= \\
<
\end{array}\right\} 0 .
$$

Let us note that the study of model $(A)$ involves many different cases requiring rather distinct types of analysis. When $n=2$, the analysis of model $(A)$ belongs more to the field of ordinal measurement than to that of conjoint measurement (we have $x \in \mathscr{A} \Leftrightarrow v_{1}\left(x_{1}\right)>-v_{2}\left(x_{2}\right)$, which is quite reminiscent of ordinal measurement). This case is tackled in Bouyssou and Marchant (2008b) using extensions of classical results on biorders (Doignon et al., 1984). In the same vein, the case in which $X$ is finite involves the use of standard separation techniques from Linear Algebra. It is analyzed in Bouyssou and Marchant (2008a). We concentrate here on the case in which $n \geq 3$ and the set of alternatives has a "rich" structure, which is obtained using solvability assumptions. We will show that it is possible to adapt in a simple way the classical results of conjoint measurement characterizing the additive value function model (see Krantz et al., 1971, Ch. 6) to analyze model $(A)$. The price to pay for such a simple adaptation will be the need for strong solvability assumptions. In a companion paper (Bouyssou and Marchant, 2009), we consider weaker solvability assumptions that lead to results that are more powerful but that are no more simple adaptations of classical conjoint measurement results.

\section{Intuition of the axioms and sketch of an as- sessment protocol}

We want to build real-valued functions $v_{i}$ on $X_{i}$ such that model $(A)$ holds through a structured dialogue with the DM. In this dialogue, several alternatives will be presented to him/her and he/she is expected to answer indicating to which category in $\langle\mathscr{A}, \mathscr{F}, \mathscr{U}\rangle$ these alternatives belong. No other type of questions will be asked to the DM. The aim of this section is to give a rough sketch of an assessment 
protocol of the an additive value function model representing an ordered partition $\langle\mathscr{A}, \mathscr{F}, \mathscr{U}\rangle$. This will also be the occasion to informally motivate the various conditions that we will later use. Formal definitions will be given in Section 4 .

\subsection{Ordering the elements of $X_{i}$ and compatibility}

An obvious consequence of model $(A)$ is that the function $v_{i}$ induces a weak ordering (i.e., a complete and transitive binary relation) on the elements of $X_{i}$ letting, for all $x_{i}, y_{i} \in X_{i}$,

$$
x_{i} \succsim_{i} y_{i} \Leftrightarrow v_{i}\left(x_{i}\right) \geq v_{i}\left(y_{i}\right)
$$

This weak order $\succsim_{i}$ is compatible with the partition $\langle\mathscr{A}, \mathscr{F}, \mathscr{U}\rangle$ in the following sense. If $x_{i} \succsim_{i} y_{i}$ and $\left(y_{i}, a_{-i}\right) \in \mathscr{A}$ it follows that $\left(x_{i}, a_{-i}\right) \in \mathscr{A}$. Moreover, if $x_{i} \succsim_{i} y_{i}$ and $\left(y_{i}, a_{-i}\right) \in \mathscr{F}$ it follows that $\left(x_{i}, a_{-i}\right) \in \mathscr{A} \mathscr{F}$. The linearity condition introduced below will ensure that it is possible to define a weak order on each $X_{i}$ that is compatible with $\langle\mathscr{A}, \mathscr{F}, \mathscr{U}\rangle$ on all $i \in N$. It excludes, e.g., the existence of $x_{i}, y_{i} \in X_{i}$ and $a_{-i}, b_{-i} \in X_{-i}$ such that $\left(x_{i}, a_{-i}\right) \in \mathscr{A},\left(y_{i}, b_{-i}\right) \in \mathscr{A},\left(y_{i}, a_{-i}\right) \in$ $\mathscr{F} \mathscr{U},\left(x_{i}, b_{-i}\right) \in \mathscr{F} \mathscr{U}$. Indeed, such a configuration is clearly incompatible with model $(A)$ since the first and the third relation imply $v_{i}\left(x_{i}\right)>v_{i}\left(y_{i}\right)$, while the second and the fourth imply $v_{i}\left(y_{i}\right)>v_{i}\left(x_{i}\right)$.

Observe that, in fact, model $(A)$ require more. For all nonempty proper subset $I$ of $N$, we should be in position de define a weak order $\succsim_{I}$ that is compatible with $\langle\mathscr{A}, \mathscr{F}, \mathscr{U}\rangle$. This is what we will call below strong linearity.

\subsection{Strict compatibility}

We have just shown how we can define a weak order $\succsim_{i}$ on each $X_{i}$ that is compatible with $\langle\mathscr{A}, \mathscr{F}, \mathscr{U}\rangle$. We now pay attention to category $\mathscr{F}$ that plays in model $(A)$ the role of a frontier between $\mathscr{A}$ and $\mathscr{U}$. Suppose that we know that $\left(x_{i}, a_{-i}\right) \in \mathscr{F}$ and $\left(y_{i}, a_{-i}\right) \in \mathscr{F}$. Clearly model $(A)$ implies that we must have $v_{i}\left(x_{i}\right)=v_{i}\left(y_{i}\right)$. This implies that, whatever $b_{-i} \in X_{-i}$, it is impossible that $\left(x_{i}, b_{-i}\right)$ and $\left(y_{i}, b_{-i}\right)$ belong to different categories in $\langle\mathscr{A}, \mathscr{F}, \mathscr{U}\rangle$. This motivates the thinness condition introduced below that captures the fact that category $\mathscr{F}$ is indeed special in our 
model. This condition would not hold in a model such that, for all $x, y \in X$,

$$
\begin{aligned}
& x \in \mathscr{A} \Leftrightarrow \sum_{i=1}^{n} v_{i}\left(x_{i}\right)>1, \\
& x \in \mathscr{F} \Leftrightarrow-1 \leq \sum_{i=1}^{n} v_{i}\left(x_{i}\right) \leq 1, \\
& x \in \mathscr{U} \Leftrightarrow \sum_{i=1}^{n} v_{i}\left(x_{i}\right)<-1 .
\end{aligned}
$$

An easy but important consequence of adding thinness to linearity is that if $\left(y_{i}, a_{-i}\right) \in \mathscr{F}$ and $x_{i} \succ_{i} y_{i}$, we must have $\left(x_{i}, a_{-i}\right) \in \mathscr{A}$. Similarly, $\left(y_{i}, a_{-i}\right) \in \mathscr{F}$ and $y_{i} \succ_{i} z_{i}$ imply $\left(z_{i}, a_{-i}\right) \in \mathscr{U}$. These additional conditions lead to what we call strict compatibility between $\succsim_{i}$ and $\langle\mathscr{A}, \mathscr{F}, \mathscr{U}\rangle$.

Finally as in the case of linearity, model $(A)$ requires that we should have a similar condition for all nonempty proper subset $I$ of $N$. This is what we will call below strong thinness.

At this stage, we know that if linearity and thinness (resp. strong linearity and strong thinness) hold, there will be a weak order on each $X_{i}$ (resp. $X_{I}$ ) that is strictly compatible with $\langle\mathscr{A}, \mathscr{F}, \mathscr{U}\rangle$.

\subsection{Uniqueness}

Suppose that there are real-valued functions $v_{i}$ on $X_{i}$ such that model $(A)$ holds. It is clear that these functions are not unique. For instance, let $u_{i}=\alpha v_{i}+\beta_{i}$ with $\alpha>0$ and $\sum_{i=1}^{n} \beta_{i}=0$. It is easy to check that the functions $u_{i}$ also represent $\langle\mathscr{A}, \mathscr{F}, \mathscr{U}\rangle$ in model $(A)$. This means that we can arbitrarily choose the "unit of measurement" in our model (this corresponds to the parameter $\alpha$ ) and that we can always arbitrarily choose the "origin of measurement" (this corresponds to the parameters $\beta_{i}$ ). We will later see that, when the set of alternatives is rich, these are the only transformations that can be applied to the functions $v_{i}$.

\subsection{Conventions and normalization}

We suppose till Section 3.8 that $n=3$. This will turn out to be the most difficult case. Hence, we have $X=X_{1} \times X_{2} \times X_{3}$. We want to build real-valued functions $v_{1}, v_{2}$ and $v_{3}$ so that model $(A)$ holds.

Suppose that we can find an alternative $\left(x_{1}^{0}, x_{2}^{0}, x_{3}^{0}\right) \in \mathscr{F}$ (in what follows superscripts are often used to differentiate elements and not to indicate exponentiation). From the above remarks on uniqueness, it is clear that we may always take $v_{1}\left(x_{1}^{0}\right)=0, v_{2}\left(x_{2}^{0}\right)=0$ and $v_{3}\left(x_{3}^{0}\right)=0$. These choice will fix the origins of the scales 
$v_{i}$. Take any element $x_{1}^{-1} \in X_{1}$ such that $x_{1}^{0} \succ_{1} x_{1}^{-1}$. Strict compatibility implies $\left(x_{1}^{-1}, x_{2}^{0}, x_{3}^{0}\right) \in \mathscr{U}$. We can always suppose that we have $v_{1}\left(x_{1}^{-1}\right)=-1$. This will fix the unit of the scale $v_{1}$ using the parameter $\alpha$ introduced above. Hence, this will also fix the unit of all scales $v_{i}$. Once these choices are made we should be in position to assess the entire functions $v_{i}$.

Notice that we have supposed that we will be able to find an object $\left(x_{1}^{0}, x_{2}^{0}, x_{3}^{0}\right)$ belonging to $\mathscr{F}$. This will result from our unrestricted solvability assumption below that will require that for all $x \in X$ and for all $i \in N$, there will be a $y_{i} \in X_{i}$ such that $\left(y_{i}, x_{-i}\right) \in \mathscr{F}$. Notice also that the above normalization requires that there are two elements $x_{1}^{0}, x_{1}^{-1} \in X_{1}$ such that $x_{1}^{0} \succ_{1} x_{1}^{-1}$. This will require that attribute 1 is influent.

\subsection{Building diagonal standard sequences}

The classical theory of conjoint measurement vitally rests on the possibility to build standard sequences on each attributes, i.e., sequences of elements that are equally spaced in the numerical model. The same will be true here. The main difference with the classical case is that the process of building standard sequences here will, in fact, lead to build equally spaced sequences on two attributes at the same time. This is what we call a diagonal standard sequence. The assessment protocol will single out one attribute and concentrate on the tradeoffs between the remaining two attributes on which we will build a diagonal standard sequence. We single out attribute 1 since it was chosen above to fix the unit of measurement in the model.

The first question to the DM aims at finding an element $x_{2}^{1} \in X_{2}$ such that $\left(x_{1}^{-1}, x_{2}^{1}, x_{3}^{0}\right) \in \mathscr{F}$. If model $(A)$ holds, we must have $v_{1}\left(x_{1}^{-1}\right)+v_{2}\left(x_{2}^{1}\right)+v_{3}\left(x_{3}^{0}\right)=0$. This implies $v_{2}\left(x_{2}^{1}\right)=1$. We next ask for a value $x_{3}^{-1} \in X_{3}$ such that $\left(x_{1}^{0}, x_{2}^{1}, x_{3}^{-1}\right) \in$ $\mathscr{F}$. This implies $v_{3}\left(x_{3}^{-1}\right)=-1$. The reader will have noticed that the questions alternate using $x_{1}^{-1}$ and $x_{1}^{0}$ as benchmarks. Hence the next question is to find $x_{2}^{2}$ such that $\left(x_{1}^{-1}, x_{2}^{2}, x_{3}^{-1}\right) \in \mathscr{F}$, implying $v_{2}\left(x_{2}^{2}\right)=2$. We then ask for $x_{3}^{-2}$ such that $\left(x_{1}^{0}, x_{2}^{2}, x_{3}^{-2}\right) \in \mathscr{F}$, implying $v_{3}\left(x_{3}^{-2}\right)=-2$. Note that this process can be iterated to define $x_{2}^{\ell}$ and $x_{3}^{-\ell}$ such that $\left(x_{1}^{-1}, x_{2}^{\ell}, x_{3}^{-\ell+1}\right) \in \mathscr{F},\left(x_{1}^{0}, x_{2}^{\ell}, x_{3}^{-\ell}\right) \in \mathscr{F}, v_{2}\left(x_{2}^{\ell}\right)=\ell$, and $v_{3}\left(x_{3}^{-\ell}\right)=-\ell$.

For the time being we have only assessed points with positive values on $X_{2}$ and points with negative values on $X_{3}$. Hence we start another round of questions to assess points with positive values on $X_{3}$ and points with negative values on $X_{2}$. This is done as follows. We first find $x_{3}^{1}$ such that $\left(x_{1}^{-1}, x_{2}^{0}, x_{3}^{1}\right) \in \mathscr{F}$, implying $v_{3}\left(x_{3}^{1}\right)=1$. We then find $x_{2}^{-1}$ such that $\left(x_{1}^{0}, x_{2}^{-1}, x_{3}^{1}\right) \in \mathscr{F}$, implying $v_{2}\left(x_{2}^{-1}\right)=-1$. The assessment process continues in the same way as before. We ask for $x_{3}^{2}$ such that $\left(x_{1}^{-1}, x_{2}^{-1}, x_{3}^{2}\right) \in \mathscr{F}$, implying $v_{3}\left(x_{3}^{2}\right)=2$. We then ask for $x_{2}^{-2}$ such that $\left(x_{1}^{0}, x_{2}^{-2}, x_{3}^{2}\right) \in \mathscr{F}$, implying $v_{2}\left(x_{2}^{-2}\right)=-2$. We will eventually find $x_{2}^{-\ell}$ and $x_{3}^{\ell}$ such 
that $\left(x_{1}^{-1}, x_{2}^{-\ell+1}, x_{3}^{\ell}\right) \in \mathscr{F}$ and $\left(x_{1}^{0}, x_{2}^{-\ell}, x_{3}^{\ell}\right) \in \mathscr{F}, v_{3}\left(x_{3}^{\ell}\right)=\ell$, and $v_{2}\left(x_{2}^{-\ell}\right)=-\ell$. This process is illustrated in Figure 1.

As in classical conjoint measurement, we have therefore built on both $X_{2}$ and $X_{3}$ sequences of elements that must be equally spaced. Clearly, it is important that these sequences can "reach" any element of $X_{2}$ and $X_{3}$. This will be the role of the Archimedean condition introduced below. It roughly says that if the elements $x_{2}^{\tau}$ for $\tau \in \mathbb{T} \subseteq \mathbb{Z}$, are equally spaced and there are $\underline{x}_{2}, \bar{x}_{2} \in X_{2}$ such that $\bar{x}_{2} \succ_{2} x_{2}^{\tau} \succ_{2} \underline{x}_{2}$ then the standard sequence must be finite, i.e., the above process will lead to define only finitely many elements of $X_{2}$ that are equally spaced. This allows us to rule out the existence of elements of $X_{2}$ that would be so desirable or so undesirable that the above process would not allow to reach them.

\subsection{The Thomsen condition}

We now have to check that the standard sequences on $X_{2}$ and $X_{3}$ built above give a sound basis for the construction of our additive representation. We must also show how to define a standard sequence on the attribute 1 that was singled out above. The clue to this step will be the Thomsen condition. It says that if $\left(a_{1}, x_{2}, x_{3}\right) \in \mathscr{F},\left(x_{2}, x_{3}\right) \sim_{23}\left(y_{2}, y_{3}\right),\left(b_{1}, y_{2}, z_{3}\right) \in \mathscr{F}$, and $\left(y_{2}, z_{3}\right) \sim_{23}\left(z_{2}, x_{3}\right)$ then we must have $\left(x_{2}, z_{3}\right) \sim_{23}\left(z_{2}, y_{3}\right)$.

This condition will allow us to extend the standard sequences to $X_{1}$ in a consistent way. Indeed, we know that $\left(x_{1}^{0}, x_{2}^{1}, x_{3}^{-1}\right) \in \mathscr{F}$ and $\left(x_{1}^{0}, x_{2}^{0}, x_{3}^{0}\right) \in \mathscr{F}$. Because of strict compatibility, this implies that $\left(x_{2}^{1}, x_{3}^{-1}\right) \sim_{23}\left(x_{2}^{0}, x_{3}^{0}\right)$. Similarly, we know that $\left(x_{1}^{-1}, x_{2}^{0}, x_{3}^{1}\right) \in \mathscr{F}$ and $\left(x_{1}^{-1}, x_{2}^{2}, x_{3}^{-1}\right) \in \mathscr{F}$. Because of strict compatibility, this implies that $\left(x_{2}^{0}, x_{3}^{1}\right) \sim_{23}\left(x_{2}^{2}, x_{3}^{-1}\right)$. Using the Thomsen condition, we obtain that $\left(x_{2}^{1}, x_{3}^{1}\right) \sim_{23}\left(x_{2}^{2}, x_{3}^{0}\right)$. Using solvability, we can find a $x_{1}^{-2}$ such that $\left(x_{1}^{-2}, x_{2}^{1}, x_{3}^{1}\right) \in \mathscr{F}$. Because $\left(x_{2}^{1}, x_{3}^{1}\right) \sim_{23}\left(x_{2}^{2}, x_{3}^{0}\right)$, we know that we also have that $\left(x_{1}^{-2}, x_{2}^{2}, x_{3}^{0}\right) \in \mathscr{F}$. This is illustrated in Figure 2 .

It is easy to see, using Figure 2, that this process can be repeated to create an entire new curve corresponding to the element $x_{1}^{-2}$. Once this is done, we can build an entire curve corresponding to $x_{1}^{-3}$ and so on.

Similarly, we can also use the Thomsen condition to build a curve corresponding to $x_{1}^{1}, x_{1}^{2}, x_{1}^{3}$, etc. For all these elements $x_{1}^{\ell}$, it is clear that we must have $v_{1}\left(x_{1}^{\ell}\right)=\ell$. For instance, we know that $\left(x_{1}^{-1}, x_{2}^{0}, x_{3}^{1}\right) \in \mathscr{F}$ and $\left(x_{1}^{-1}, x_{2}^{1}, x_{3}^{0}\right) \in \mathscr{F}$, so that $\left(x_{2}^{0}, x_{3}^{1}\right) \sim_{23}\left(x_{2}^{1}, x_{3}^{0}\right)$. We also have $\left(x_{1}^{0}, x_{2}^{1}, x_{3}^{-1}\right) \in \mathscr{F}$ and $\left(x_{1}^{0}, x_{2}^{-1}, x_{3}^{1}\right) \in \mathscr{F}$, so that $\left(x_{2}^{1}, x_{3}^{-1}\right) \sim_{23}\left(x_{2}^{-1}, x_{3}^{1}\right)$. Using the Thomsen condition implies $\left(x_{2}^{0}, x_{3}^{-1}\right) \sim_{23}$ $\left(x_{2}^{-1}, x_{3}^{0}\right)$. The level $x_{1}^{1}$ is defined in such a way that $\left(x_{1}^{1}, x_{2}^{0}, x_{3}^{-1}\right) \in \mathscr{F}$ (and, hence, $\left.\left(x_{1}^{1}, x_{2}^{-1}, x_{3}^{0}\right) \in \mathscr{F}\right)$. The reader will easily check that if we use this newly defined level $x_{1}^{1}$ to build a diagonal standard sequence on $X_{2}$ and $X_{3}$ we would obtain the same diagonal standard sequence as the one found based on $x_{1}^{-1}$.

Finally observe that using the level $x_{2}^{-1}$ (resp. $x_{3}^{-1}$ ), we can build a diagonal 


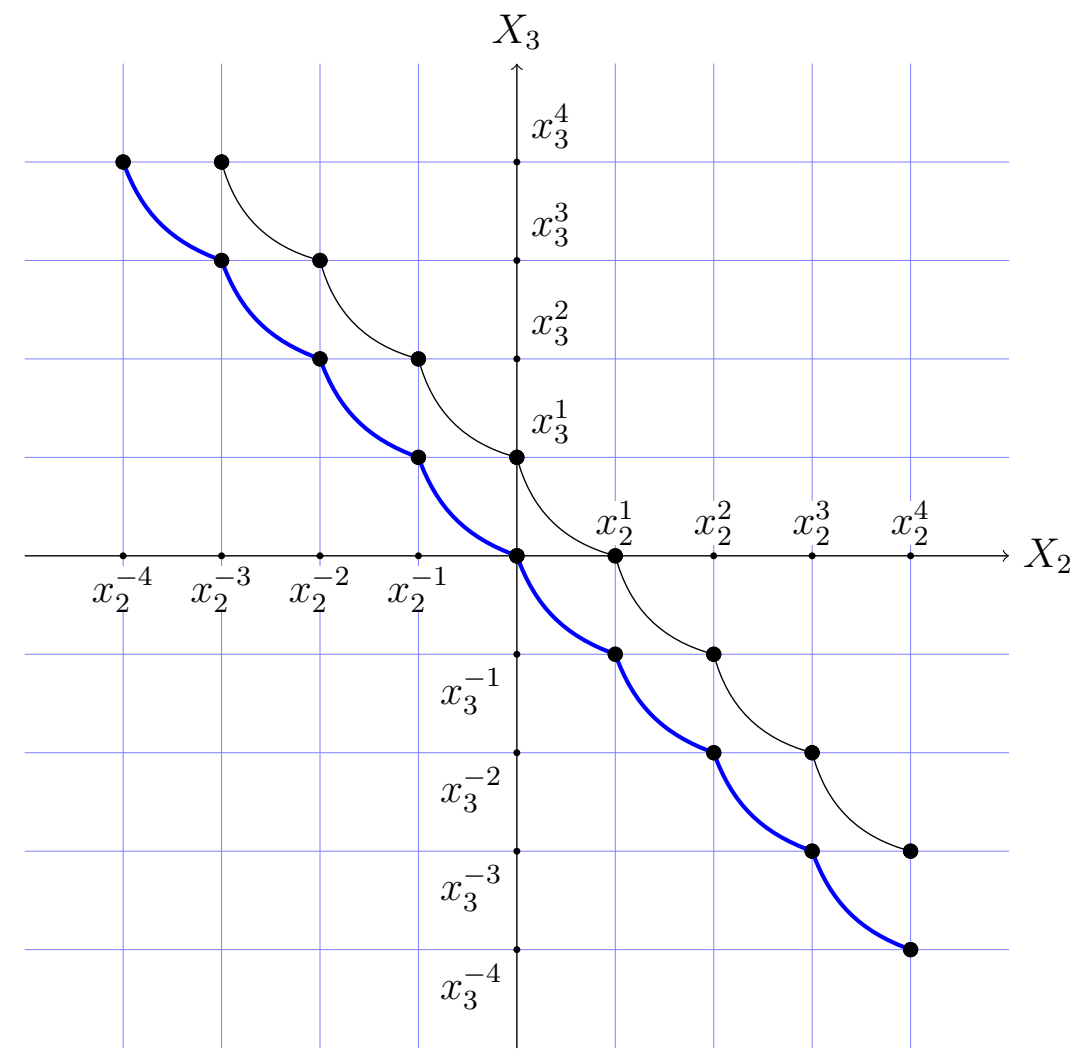

Figure 1: Building diagonal standard sequences. Points joined by a curve belong to $\mathscr{F}$ when adjoined the same element in $X_{1}\left(x_{1}^{0}\right.$ for the lower curve, $x_{1}^{-1}$ for the upper curve).

Sequence of questions:

$$
\begin{aligned}
&\left(x_{1}^{0}, x_{2}^{0}, x_{3}^{0}\right) \in \mathscr{F}, \\
&\left(x_{1}^{-1}, x_{2}^{1}, x_{3}^{0}\right) \in \mathscr{F}, \quad\left(x_{1}^{0}, x_{2}^{1}, x_{3}^{-1}\right) \in \mathscr{F}, \\
&\left(x_{1}^{-1}, x_{2}^{2}, x_{3}^{-1}\right) \in \mathscr{F}, \quad\left(x_{1}^{0}, x_{2}^{2}, x_{3}^{-2}\right) \in \mathscr{F}, \\
&\left(x_{1}^{-1}, x_{2}^{3}, x_{3}^{-2}\right) \in \mathscr{F}, \quad\left(x_{1}^{0}, x_{2}^{3}, x_{3}^{-3}\right) \in \mathscr{F}, \\
&\left(x_{1}^{-1}, x_{2}^{4}, x_{3}^{-3}\right) \in \mathscr{F}, \quad\left(x_{1}^{0}, x_{2}^{4}, x_{3}^{-4}\right) \in \mathscr{F}, \\
&\left(x_{1}^{-1}, x_{2}^{0}, x_{3}^{1}\right) \in \mathscr{F}, \quad\left(x_{1}^{0}, x_{2}^{-1}, x_{3}^{1}\right) \in \mathscr{F}, \\
&\left(x_{1}^{-1}, x_{2}^{-1}, x_{3}^{2}\right) \in \mathscr{F}, \quad\left(x_{1}^{0}, x_{2}^{-2}, x_{3}^{2}\right) \in \mathscr{F}, \\
&\left(x_{1}^{-1}, x_{2}^{-2}, x_{3}^{3}\right) \in \mathscr{F}, \quad\left(x_{1}^{0}, x_{2}^{-3}, x_{3}^{3}\right) \in \mathscr{F}, \\
&\left(x_{1}^{-1}, x_{2}^{-3}, x_{3}^{4}\right) \in \mathscr{F}, \quad\left(x_{1}^{0}, x_{2}^{-4}, x_{3}^{4}\right) \in \mathscr{F} .
\end{aligned}
$$




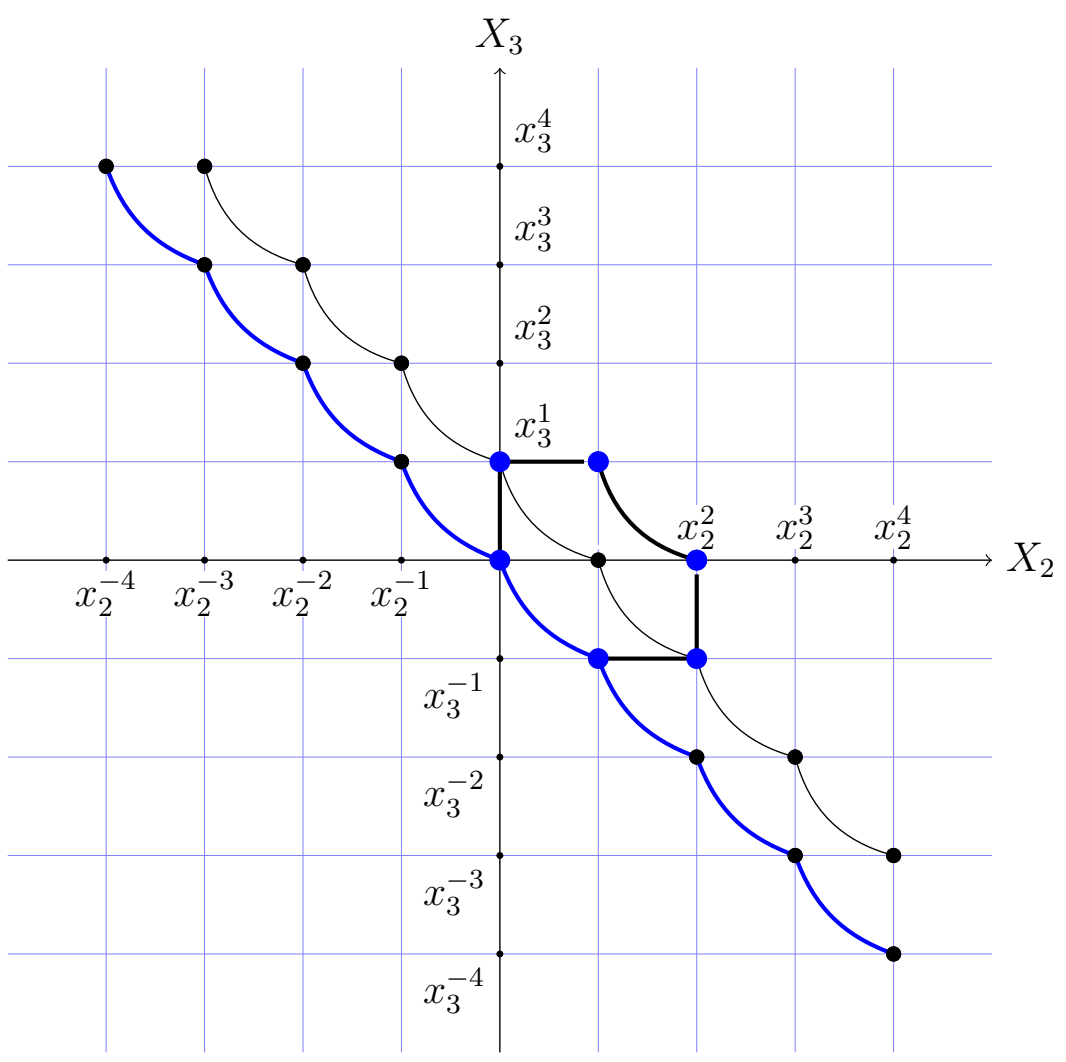

Figure 2: Using the Thomsen condition.

$$
\left.\begin{array}{l}
\left(x_{1}^{0}, x_{2}^{1}, x_{3}^{-1}\right) \in \mathscr{F},\left(x_{1}^{0}, x_{2}^{0}, x_{3}^{0}\right) \in \mathscr{F} \\
\left(x_{1}^{-1}, x_{2}^{0}, x_{3}^{1}\right) \in \mathscr{F},\left(x_{1}^{-1}, x_{2}^{2}, x_{3}^{-1}\right) \in \mathscr{F}
\end{array}\right\} \Rightarrow\left(x_{1}^{-2}, x_{2}^{1}, x_{3}^{1}\right) \in \mathscr{F},\left(x_{1}^{-2}, x_{2}^{2}, x_{3}^{0}\right) \in \mathscr{F} .
$$


standard sequence on $X_{1}$ and $X_{3}$ (resp. $X_{1}$ and $X_{2}$ ). It is simple to check that building this diagonal standard sequence will lead to define the same points $x_{1}^{k}$ and $x_{3}^{k}$ (resp. $x_{1}^{k}$ and $x_{2}^{k}$ ) as before. For instance, we would ask the DM for $y_{1}^{1}$ and $y_{3}^{1}$ such that $\left(y_{1}^{1}, x_{2}^{-1}, x_{3}^{0}\right) \in \mathscr{F}$ and $\left(x_{1}^{0}, x_{2}^{-1}, y_{3}^{-1}\right) \in \mathscr{F}$. Since we know that $\left(x_{1}^{0}, x_{2}^{-1}, x_{3}^{-1}\right) \in \mathscr{F}$, strict compatibility implies that $y_{3}^{-1} \sim_{3} x_{3}^{-1}$. Similarly, since we know that $\left(x_{1}^{1}, x_{2}^{-1}, x_{3}^{0}\right) \in \mathscr{F}$, we must have $y_{1}^{1} \sim_{1} x_{1}^{1}$.

\subsection{Checking and completing the construction}

Let us first show that the above construction is sound. On each of the attributes we have found equally spaced points $x_{i}^{k}$ and $x_{i}^{k+1}$, for $k \in \mathbb{Z}$. In any additive numerical representation that is normalized as decided above, we must have that $v_{i}\left(x_{i}^{k}\right)=k$. It remains to be shown that for all $k, \ell, m \in \mathbb{Z}$, we have $\left(x_{1}^{k}, x_{2}^{\ell}, x_{3}^{m}\right) \in \mathscr{F}$ iff $k+\ell+m=0$ and $\left(x_{1}^{k}, x_{2}^{\ell}, x_{3}^{m}\right) \in \mathscr{A}$ iff $k+\ell+m>0$. Using Figure 2, it is clear that we have, for all $k \in \mathbb{Z},\left(x_{1}^{k}, x_{2}^{-k}, x_{3}^{0}\right) \in \mathscr{F}$. Furthermore, we know that if $\left(x_{1}^{k}, x_{2}^{\ell}, x_{3}^{m}\right) \in \mathscr{F}$ then we also have $\left(x_{1}^{k}, x_{2}^{\ell+1}, x_{3}^{m-1}\right) \in \mathscr{F}$.

Consider now $\left(x_{1}^{k}, x_{2}^{\ell}, x_{3}^{m}\right)$ such that $k+\ell+m=0$, which implies that $\ell+m=$ $-k$. After having added several times 1 to $\ell$ and subtracted it to $m$, we will find an element $\left(x_{1}^{k}, x_{2}^{\ell+m}, x_{3}^{0}\right)$ such that $\ell+m=-k$. But we know that $\left(x_{1}^{k}, x_{2}^{-k}, x_{3}^{0}\right) \in \mathscr{F}$. This will imply, in view of Figure 2 , that $\left(x_{1}^{k}, x_{2}^{\ell}, x_{3}^{m}\right) \in \mathscr{F}$ when $k+\ell+m=0$. The fact that $\left(x_{1}^{k}, x_{2}^{\ell}, x_{3}^{m}\right) \in \mathscr{A}$ when $k+\ell+m>0$ is easily shown. Since we always have $x_{i}^{k} \succ_{i} x_{i}^{k-1}$, the desired conclusion follows from the fact that all relations $\succsim_{i}$ are strictly compatible with $\langle\mathscr{A}, \mathscr{F}, \mathscr{U}\rangle$. Hence, our construction of equally spaced points on each $X_{i}$ is sound.

Let us briefly show how it is possible to extend this construction to all points of $X_{i}$. We do so for $X_{2}$. Suppose for definiteness that $y_{2} \succ_{2} x_{2}^{1}$. Our aim is to assess $v_{2}\left(y_{2}\right)$. The process is simple. Using solvability, we find an element $y_{1}^{-1} \in X_{1}$ such that $\left(y_{1}^{-1}, y_{2}, x_{3}^{0}\right) \in \mathscr{F}$. We then use this value $y_{1}^{-1}$ to build a diagonal standard sequence on $X_{2}$ and $X_{3}$. Consider the elements of the diagonal standard belonging to $X_{2}$ and label these elements $y_{2}^{1}=y_{2}, y_{2}^{2}, y_{2}^{3}$, and so on. It is easy to see that each element $y_{2}^{k}$ of this new standard sequence will lie in between two elements of the original standard sequence, i.e., for all $k$ such that $y_{2}^{k}$ is an element of the new standard sequence, there are elements $x_{2}^{\ell}$ and $x_{2}^{\ell+1}$ of the original standard sequence such that $x_{2}^{\ell} \succsim_{2} y_{2}^{k} \succsim_{2} x_{2}^{\ell+1}$. We know that $v_{2}\left(x_{2}^{\ell}\right)=\ell$ and $v_{2}\left(x_{2}^{\ell+1}\right)=\ell+1$. Furthermore, it must be true that $v_{2}\left(y_{2}^{k}\right)=k v_{2}\left(y_{2}\right)$, because $y_{2}^{1}=y_{2}, y_{2}^{2}, y_{2}^{3}$ is a standard sequence. Hence we have $\ell / k \leq v_{2}\left(y_{2}\right) \leq(\ell+1) / k$. This gives an easy way to approximate $v_{2}\left(y_{2}\right)$. Increasing $k$ will make this approximation more and more precise. At the limit, this will give a precise value for $v_{2}\left(y_{2}\right)$. 


\subsection{At least four attributes}

The process outlined above to assess an additive value when there are three attributes can be conducted in a similar way when there are four attributes or more. The main difference in this case will be that it will not be necessary to explicitly invoke the Thomsen condition It will be implied by the conjunction of our other conditions ${ }^{1}$. Intuitively, when there are three attributes, saying that linearity holds on each attribute implies strong linearity. Indeed ordering the elements of $X_{i}$ implies ordering the elements in $X_{-i}$, so that linearity on all attributes implies linearity of all proper subsets of attributes. This is no more true when there are four attributes. If the elements of $X_{1}$ are ordered, this will imply that the elements in $X_{2} \times X_{3} \times X_{4}$ are ordered but not necessarily that the elements in $X_{3} \times X_{4}$ are ordered. This shows that strong linearity implies more than just linearity when there are four attributes or more.

Let us briefly illustrate the assessment process when $n=4$. We start with an alternative $\left(x_{1}^{0}, x_{2}^{0}, x_{3}^{0}, x_{4}^{0}\right) \in \mathscr{F}$. We may always take $v_{1}\left(x_{1}^{0}\right)=0, v_{2}\left(x_{2}^{0}\right)=0$, $v_{3}\left(x_{3}^{0}\right)=0$, and $v_{4}\left(x_{4}^{0}\right)=0$. Take any element $x_{4}^{-1} \in X_{4}$ such that $x_{4}^{0} \succ_{1} x_{4}^{-1}$. We normalize $v_{4}$ in such a way that $v_{4}\left(x_{4}^{-1}\right)=-1$.

Using the level $x_{4}^{-1}$, we can build a diagonal standard sequence on $X_{1}$ and $X_{2}$. Such a process will lead, in particular, to define the points $x_{1}^{1}, x_{2}^{-1}, x_{1}^{2}$ and $x_{2}^{-2}$ such that $\left(x_{1}^{1}, x_{2}^{0}, x_{3}^{0}, x_{4}^{-1}\right) \in \mathscr{F},\left(x_{1}^{1}, x_{2}^{-1}, x_{3}^{0}, x_{4}^{0}\right) \in \mathscr{F},\left(x_{1}^{2}, x_{2}^{-1}, x_{3}^{0}, x_{4}^{-1}\right) \in \mathscr{F}$, $\left(x_{1}^{2}, x_{2}^{-2}, x_{3}^{0}, x_{4}^{0}\right) \in \mathscr{F}$.

Similarly, using $x_{4}^{-1}$ we can build a diagonal standard sequence on $X_{1}$ and $X_{3}$. Such a process will lead to define the points $y_{1}^{1}, x_{3}^{-1}, y_{1}^{2}$ and $x_{3}^{-2}$ such that $\left(y_{1}^{1}, x_{2}^{0}, x_{3}^{0}, x_{4}^{-1}\right) \in \mathscr{F},\left(y_{1}^{1}, x_{2}^{0}, x_{3}^{-1}, x_{4}^{0}\right) \in \mathscr{F},\left(y_{1}^{2}, x_{2}^{0}, x_{3}^{-1}, x_{4}^{-1}\right) \in \mathscr{F},\left(y_{1}^{2}, x_{2}^{0}, x_{3}^{-2}, x_{4}^{0}\right) \in$ $\mathscr{F}$.

Because we have $\left(x_{1}^{1}, x_{2}^{0}, x_{3}^{0}, x_{4}^{-1}\right) \in \mathscr{F}$ and $\left(y_{1}^{1}, x_{2}^{0}, x_{3}^{0}, x_{4}^{-1}\right) \in \mathscr{F}$, we must have $y_{1}^{1} \sim_{1} x_{1}^{1}$. Let us check that we also have $y_{1}^{2} \sim_{1} x_{1}^{2}$. Indeed, we know that $\left(x_{1}^{1}, x_{2}^{-1}, x_{3}^{0}, x_{4}^{0}\right) \in \mathscr{F}$ and $\left(x_{1}^{1}, x_{2}^{0}, x_{3}^{-1}, x_{4}^{0}\right) \in \mathscr{F}$. This implies that $\left(x_{2}^{-1}, x_{3}^{0}\right) \sim_{23}$ $\left(x_{2}^{0}, x_{3}^{-1}\right)$. Hence $\left(x_{1}^{2}, x_{2}^{-1}, x_{3}^{0}, x_{4}^{-1}\right) \in \mathscr{F}$ implies $\left(x_{1}^{2}, x_{2}^{0}, x_{3}^{-1}, x_{4}^{-1}\right) \in \mathscr{F}$. Since we know that $\left(y_{1}^{2}, x_{2}^{0}, x_{3}^{-1}, x_{4}^{-1}\right) \in \mathscr{F}$, we obtain $y_{1}^{2} \sim_{1} x_{1}^{2}$. This reasoning is easily extended to show that all points $x_{1}^{k}$ defined building a diagonal standard sequence on $X_{1}$ and $X_{2}$ and all points $y_{1}^{k}$ defined building a diagonal standard sequence on $X_{1}$ and $X_{3}$ will coincide.

Using a similar reasoning shows that the process of building diagonal standard sequences will never lead to contradictions. The completion of the construction is obtained as before, using the Thomsen condition.

Let us outline the proof of the fact that the Thomsen condition will hold when $n \geq 4$. Suppose that $\left(x_{1}, x_{2}, a_{3}, a_{4}\right) \in \mathscr{F},\left(x_{1}, x_{2}\right) \sim_{12}\left(y_{1}, y_{2}\right),\left(y_{1}, z_{2}, b_{3}, b_{4}\right) \in \mathscr{F}$,

\footnotetext{
${ }^{1} \mathrm{~A}$ similar effect occurs in classical conjoint measurement: the Thomsen condition has there only to be invoked when there are two attributes
} 
and $\left(y_{1}, z_{2}\right) \sim_{12}\left(z_{1}, x_{2}\right)$. We must show that we have $\left(x_{1}, z_{2}\right) \sim_{12}\left(z_{1}, y_{2}\right)$.

Let $c_{4} \in X_{4}$. Using solvability, we will be able to find $c_{3}, d_{3} \in X_{3}$ such that $\left(x_{1}, x_{2}, c_{3}, c_{4}\right) \in \mathscr{F}$, and $\left(y_{1}, x_{2}, d_{3}, c_{4}\right) \in \mathscr{F}$. This implies $\left(x_{1}, c_{3}\right) \sim_{13}$ $\left(y_{1}, d_{3}\right)$. Since $\left(x_{1}, x_{2}\right) \sim_{12}\left(y_{1}, y_{2}\right)$, we also have $\left(y_{1}, y_{2}, c_{3}, c_{4}\right) \in \mathscr{F}$. In view of $\left(y_{1}, x_{2}, d_{3}, c_{4}\right) \in \mathscr{F}$ and $\left(y_{1}, y_{2}, c_{3}, c_{4}\right) \in \mathscr{F}$, we obtain $\left(x_{2}, d_{3}\right) \sim_{23}\left(y_{2}, c_{3}\right)$.

Using solvability, we can find $d_{4} \in X_{4}$ such that $\left(z_{1}, x_{2}, d_{3}, d_{4}\right) \in \mathscr{F}$. Since $\left(x_{2}, d_{3}\right) \sim_{23}\left(y_{2}, c_{3}\right)$, we know that $\left(z_{1}, y_{2}, c_{3}, d_{4}\right) \in \mathscr{F}$. Similarly, since $\left(y_{1}, z_{2}\right) \sim_{12}$ $\left(z_{1}, x_{2}\right),\left(z_{1}, x_{2}, d_{3}, d_{4}\right) \in \mathscr{F}$ implies $\left(y_{1}, z_{2}, d_{3}, d_{4}\right) \in \mathscr{F}$. Since we know that $\left(y_{1}, z_{2}, d_{3}, d_{4}\right) \in \mathscr{F}$ and $\left(x_{1}, c_{3}\right) \sim_{13}\left(y_{1}, d_{3}\right)$, we obtain $\left(x_{1}, z_{2}, c_{3}, d_{4}\right) \in \mathscr{F}$. Hence, we have $\left(x_{1}, z_{2}, c_{3}, d_{4}\right) \in \mathscr{F}$ and $\left(z_{1}, y_{2}, c_{3}, d_{4}\right) \in \mathscr{F}$, which implies $\left(x_{1}, z_{2}\right) \sim_{12}$ $\left(z_{1}, y_{2}\right)$, as required.

\section{Axioms and Results}

Our aim in this section is to present conditions ensuring the existence of an additive representation of $\langle\mathscr{A}, \mathscr{F}, \mathscr{U}\rangle$ when there are at least three attributes. Starting with $\langle\mathscr{A}, \mathscr{F}, \mathscr{U}\rangle$ on $X=X_{1} \times \ldots X_{2} \times \cdots \times X_{n}$, our strategy will be to build a binary relation on a product set that leaves out one attribute, i.e., on a set $\prod_{i \neq j} X_{i}$. We will impose conditions on $\langle\mathscr{A}, \mathscr{F}, \mathscr{U}\rangle$ ensuring that this binary relation satisfies the standard axioms of conjoint measurement as given in Krantz et al. (1971, Ch. 6). This ensures the existence of an additive representation of the binary relation. Bringing the attribute that was left out in the construction of the binary relation back into the picture again, we will show that the additive representation of the binary relation can be used to obtain an additive representation of the ordered partition $\langle\mathscr{A}, \mathscr{F}, \mathscr{U}\rangle$.

\subsection{Necessary axioms}

Given any nonempty proper subset $I$ of $N$, our first condition amounts to requiring that it is possible to weakly order the elements of $X_{I}$ in such a way that this weak order is compatible with $\langle\mathscr{A}, \mathscr{F}, \mathscr{U}\rangle$.

\section{Definition 2 (Linearity)}

Let $I$ be a nonempty proper subset of $N$. We say that $\langle\mathscr{A}, \mathscr{F}, \mathscr{U}\rangle$ is

1. $\mathscr{A}$-linear on $I$ (condition $\mathscr{A}$-linear $I$ ) if

$$
\left.\begin{array}{c}
\left(x_{I}, a_{-I}\right) \in \mathscr{A} \\
\text { and } \\
\left(y_{I}, b_{-I}\right) \in \mathscr{A}
\end{array}\right\} \Rightarrow\left\{\begin{array}{c}
\left(y_{I}, a_{-I}\right) \in \mathscr{A} \\
\text { or } \\
\left(x_{I}, b_{-I}\right) \in \mathscr{A}
\end{array} \quad\left(\mathscr{A}-\operatorname{linear}_{I}\right)\right.
$$


2. $\mathscr{F}$-linear on $I$ (condition $\mathscr{F}$-linear $)$ ) if

$$
\left.\begin{array}{c}
\left(x_{I}, a_{-I}\right) \in \mathscr{F} \\
\text { and } \\
\left(y_{I}, b_{-I}\right) \in \mathscr{F}
\end{array}\right\} \Rightarrow\left\{\begin{array}{c}
\left(y_{I}, a_{-I}\right) \in \mathscr{A} \mathscr{F} \\
\text { or } \\
\left(x_{I}, b_{-I}\right) \in \mathscr{A} \mathscr{F}
\end{array}\right.
$$

3. $\mathscr{A F}$-linear on I (condition $\mathscr{A} \mathscr{F}$-linear ${ }_{I}$ ) if

$$
\left.\begin{array}{c}
\left(x_{I}, a_{-I}\right) \in \mathscr{A} \\
\text { and } \\
\left(y_{I}, b_{-I}\right) \in \mathscr{F}
\end{array}\right\} \Rightarrow\left\{\begin{array}{c}
\left(y_{I}, a_{-I}\right) \in \mathscr{A} \\
\text { or } \\
\left(x_{I}, b_{-I}\right) \in \mathscr{A} \mathscr{F}
\end{array}\right.
$$

for all $x_{I}, y_{I} \in X_{I}$ and $a_{-I}, b_{-I} \in X_{-I}$. We say that $\langle\mathscr{A}, \mathscr{F}, \mathscr{U}\rangle$ is linear $_{I}$ if it is $\mathscr{A}$-linear, $\mathscr{F}$-linear ${ }_{I}$ and $\mathscr{A} \mathscr{F}$-linear. . We say that $\langle\mathscr{A}, \mathscr{F}, \mathscr{U}\rangle$ is strongly linear if it satisfies linear, , for all nonempty proper subset $I$ of $N$.

It is easy to check that the existence of an additive representation implies strong linearity. For instance, suppose that $\left(x_{I}, a_{-I}\right) \in \mathscr{A}$ and $\left(y_{I}, b_{-I}\right) \in \mathscr{A}$, so that $\sum_{i \in I} v_{i}\left(x_{i}\right)+\sum_{j \notin I} v_{j}\left(a_{j}\right)>0$ and $\sum_{i \in I} v_{i}\left(y_{i}\right)+\sum_{j \notin I} v_{j}\left(b_{j}\right)>0$. If $\sum_{i \in I} v_{i}\left(x_{i}\right) \geq$ $\sum_{i \in I} v_{i}\left(y_{i}\right)$, we have $\sum_{i \in I} v_{i}\left(x_{i}\right)+\sum_{j \notin I} v_{j}\left(b_{j}\right)>0$, so that $\left(x_{I}, b_{-I}\right) \in \mathscr{A}$. Otherwise, we have $\sum_{i \in I} v_{i}\left(y_{i}\right)>\sum_{i \in I} v_{i}\left(x_{i}\right)$, which implies $\sum_{i \in I} v_{i}\left(y_{i}\right)+\sum_{j \notin I} v_{j}\left(a_{j}\right)>$ 0 and $\left(y_{I}, a_{-I}\right) \in \mathscr{A}$.

It is easy to check that if an ordered partition satisfies linear ${ }_{I}$ then it will satisfy a similar condition in which all instances of $\mathscr{A}$ (resp. $\mathscr{A} \mathscr{F}$ ) are replaced by $\mathscr{U}$ (resp. $\mathscr{F} \mathscr{U})$. This shows that this condition deals with $\mathscr{A}$ and $\mathscr{U}$ in a symmetric way.

The consequences of our linearity conditions can be clearly understood considering the trace that $\langle\mathscr{A}, \mathscr{F}, \mathscr{U}\rangle$ generates on each $X_{I}$. Let $I$ be a nonempty proper subset of $N$. We define on $X_{I}$ the binary relation $\succsim_{I}$ letting, for all $x_{I}, y_{I} \in X_{I}$,

$$
x_{I} \succsim_{I} y_{I} \Leftrightarrow \text { for all } a_{-I} \in X_{-I},\left\{\begin{array}{l}
\left(y_{I}, a_{-I}\right) \in \mathscr{A} \Rightarrow\left(x_{I}, a_{-I}\right) \in \mathscr{A}, \\
\left(y_{I}, a_{-I}\right) \in \mathscr{F} \Rightarrow\left(x_{I}, a_{-I}\right) \in \mathscr{A} \mathscr{F} .
\end{array}\right.
$$

We say that $\succsim_{I}$ is the trace on $X_{I}$ generated by $\langle\mathscr{A}, \mathscr{F}, \mathscr{U}\rangle$. By construction, $\succsim_{I}$ is always reflexive and transitive. We use $\succ_{I}$ and $\sim_{I}$ as is usual. The role of linear $_{I}$ is precisely to ensure that the trace $\succsim_{I}$ is complete, so that it becomes a weak order. By construction, this relation is compatible with $\langle\mathscr{A}, \mathscr{F}, \mathscr{U}\rangle$. This is summarized in the following lemma. We omit its simple proof.

\section{Lemma 1}

For all $x, y \in X$ and all nonempty proper subset $I$ of $N$, we have:

$$
\begin{aligned}
{\left[y \in \mathscr{A} \text { and } x_{I} \succsim_{I} y_{I}\right] } & \Rightarrow\left(x_{I}, y_{-I}\right) \in \mathscr{A}, \\
{\left[y \in \mathscr{F} \text { and } x_{I} \succsim_{I} y_{I}\right] } & \Rightarrow\left(x_{I}, y_{-I}\right) \in \mathscr{A} \mathscr{F}, \\
{\left[x_{i} \succsim_{i} y_{i}, \text { for all } i \in I\right] } & \Rightarrow\left[x_{I} \succsim_{I} y_{I}\right] .
\end{aligned}
$$

Furthermore, a threefold partition $\langle\mathscr{A}, \mathscr{F}, \mathscr{U}\rangle$ is linear $_{I}$ iff $\succsim_{I}$ is complete. 
As explained in Section 3.2, our next condition aims at capturing the special role played by category $\mathscr{F}$.

\section{Definition 3 (Thinness)}

Let $I$ be a nonempty proper subset of $N$. We say that the threefold partition $\langle\mathscr{A}, \mathscr{F}, \mathscr{U}\rangle$ is thin on $I$ (condition thin ${ }_{I}$ ) if,

$$
\left.\begin{array}{c}
\left(x_{I}, a_{-I}\right) \in \mathscr{F} \\
\text { and } \\
\left(y_{I}, a_{-I}\right) \in \mathscr{F}
\end{array}\right\} \Rightarrow\left\{\begin{array}{l}
\left(x_{I}, b_{-I}\right) \in \mathscr{A} \Leftrightarrow\left(y_{I}, b_{-I}\right) \in \mathscr{A}, \\
\left(x_{I}, b_{-I}\right) \in \mathscr{U} \Leftrightarrow\left(y_{I}, b_{-I}\right) \in \mathscr{U}
\end{array}\right.
$$

for all $x_{I}, y_{I} \in X_{I}$ and $a_{-I}, b_{-I} \in X_{-I}$. We say that $\langle\mathscr{A}, \mathscr{F}, \mathscr{U}\rangle$ is strongly thin if

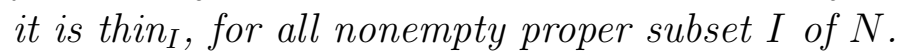

It is easy to check that the existence of an additive representation implies that the partition must be strongly thin. Indeed, if $\left(x_{I}, a_{-I}\right) \in \mathscr{F}$ and $\left(y_{I}, a_{-I}\right) \in$ $\mathscr{F}$, we must have that $\sum_{i \in I} v_{i}\left(x_{i}\right)=\sum_{i \in I} v_{i}\left(y_{i}\right)$, so that replacing $x_{I}$ by $y_{I}$ will never modify the assignment of an alternative. As discussed in Section 3.2, the combination of linear ${ }_{I}$ and $\operatorname{thin}_{I}$ will ensure that the weak order $\succsim_{I}$ becomes strictly compatible with $\langle\mathscr{A}, \mathscr{F}, \mathscr{U}\rangle$. This is summarized in the following lemma, which obvious proof is omitted.

\section{Lemma 2}

Suppose that $\langle\mathscr{A}, \mathscr{F}, \mathscr{U}\rangle$ is linear $_{I}$ and thin I $_{I}$ on a nonempty proper subset I of $N$. Then

$$
\begin{aligned}
& {\left[\left(x_{I}, a_{-I}\right) \in \mathscr{F} \text { and } y_{I} \succ_{I} x_{I}\right] \Rightarrow\left(y_{I}, a_{-I}\right) \in \mathscr{A},} \\
& {\left[\left(x_{I}, a_{-I}\right) \in \mathscr{F} \text { and } x_{I} \succ_{I} z_{I}\right] \Rightarrow\left(z_{I}, a_{-I}\right) \in \mathscr{U},}
\end{aligned}
$$

for all $x_{I}, y_{I}, z_{I} \in X_{I}$ and $a_{-I} \in X_{-I}$.

It is easy to build examples showing that condition thin $_{I}$ is, in general, independent from $\mathscr{A}$-linear ${ }_{I}, \mathscr{F}$-linear $I$ and $\mathscr{A} \mathscr{F}$-linear ${ }_{I}$.

The next condition will only come into play when $n=3$. Its importance for building additive representations was stressed in Section 3.6.

\section{Definition 4 (Thomsen condition)}

We say that $\langle\mathscr{A}, \mathscr{F}, \mathscr{U}\rangle$ on $X$ satisfies the Thomsen condition if

$$
\left.\begin{array}{r}
\left(x_{i}, x_{j}, a_{-i j}\right) \in \mathscr{F} \text { and }\left(x_{i}, x_{j}\right) \sim_{i j}\left(y_{i}, y_{j}\right) \\
\left(y_{i}, z_{j}, b_{-i j}\right) \in \mathscr{F} \text { and }\left(y_{i}, z_{j}\right) \sim_{i j}\left(z_{i}, x_{j}\right)
\end{array}\right\} \Rightarrow\left(x_{i}, z_{j}\right) \sim_{i j}\left(z_{i}, y_{j}\right)
$$

for all $i, j \in N$ with $i \neq j$, all $x_{i}, y_{i}, z_{i} \in X_{i}$, all $x_{j}, y_{j}, z_{j} \in X_{j}$ and all $a_{-i j}, b_{-i j} \in$ $X_{-i j}$. 
Let us show that this condition is necessary for model $(A)$. Indeed, $\left(x_{i}, x_{j}, a_{-i l}\right) \in$ $\mathscr{F}$ and $\left(x_{i}, x_{j}\right) \sim_{i j}\left(y_{i}, y_{j}\right)$ imply that $\left(y_{i}, y_{j}, a_{-i l}\right) \in \mathscr{F}$. This implies $v_{i}\left(x_{i}\right)+$ $v_{j}\left(x_{j}\right)=v_{i}\left(y_{i}\right)+v_{j}\left(y_{j}\right)$. Similarly, $\left(y_{i}, z_{j}, b_{-i l}\right) \in \mathscr{F}$ and $\left(y_{i}, z_{j}\right) \sim_{i j}\left(z_{i}, x_{j}\right)$ lead to $v_{i}\left(y_{i}\right)+v_{j}\left(z_{j}\right)=v_{i}\left(z_{i}\right)+v_{j}\left(x_{j}\right)$. Hence, we have $v_{i}\left(z_{i}\right)+v_{j}\left(y_{j}\right)=v_{i}\left(x_{i}\right)+v_{j}\left(z_{j}\right)$, so that $\left(x_{i}, z_{j}\right) \sim_{i j}\left(z_{i}, y_{j}\right)$. We have shown in Section 3.6 that this condition is central in the construction of an additive representation when there are three attributes.

Our next condition is a possible formalization of diagonal standard sequences and an Archimedean condition for ordered partitions. This formalization follows the construction presented above in Section 3.5.

\section{Definition 5 (Diagonal standard sequences)}

Let $i, j \in N$ with $i \neq j$. Let $\mathbb{T}$ be any set (finite or infinite) of consecutive integers (positive or negative). We say that $\left\{x_{i}^{\tau} \in X_{i}, x_{j}^{\tau} \in X_{j},: \tau \in \mathbb{T}\right\}$ is a diagonal standard sequence for $\langle\mathscr{A}, \mathscr{F}, \mathscr{U}\rangle$ on attributes $i, j \in N$ if there are $a_{-i j}, b_{-i j} \in X_{-i j}$ such that $\operatorname{Not}\left[a_{-i j} \sim_{-i j}, b_{-i j}\right]$ (i.e., there are $a_{i} \in X_{i}$ and $a_{j} \in X_{j}$ such that $\left(a_{i}, a_{j}, a_{-i j}\right)$ and $\left(a_{i}, a_{j}, b_{-i j}\right)$ do not belong to the same category) and

$$
\begin{gathered}
\left(x_{i}^{\tau}, x_{j}^{\tau-1}, b_{-i j}\right) \in \mathscr{F}, \\
\left(x_{i}^{\tau}, x_{j}^{\tau}, a_{-i j}\right) \in \mathscr{F}, \\
\left(x_{i}^{\tau+1}, x_{j}^{\tau}, b_{-i j}\right) \in \mathscr{F},
\end{gathered}
$$

for all $\tau \in \mathbb{T}$.

In Figure 1 above, we have illustrated a diagonal standard sequence on $X_{2}$ and $X_{3}$. The role of $a_{-i j}, b_{-i j} \in X_{-i j}$ is played by the two elements $x_{1}^{0}$ and $x_{1}^{-1}$. It is clear that if the sequence on $i$ is increasing (i.e., $x_{i}^{\tau+1} \succ_{i} x_{i}^{\tau}$ ) then the sequence on $j$ is decreasing (i.e., $x_{j}^{\tau-1} \succ_{j} x_{j}^{\tau}$ ) and vice versa.

Suppose that $\left\{x_{i}^{\tau} \in X_{i}, x_{j}^{\tau} \in X_{j},: \tau \in \mathbb{T}\right\}$, is a diagonal standard sequence on attributes $i$ and $j$. Because we have supposed that $N o t\left[a_{-i j} \sim_{-i j}, b_{-i j}\right]$, in any additive representation $\left\langle v_{i}\right\rangle_{i \in N}$ of $\langle\mathscr{A}, \mathscr{F}, \mathscr{U}\rangle$, we must have

$$
\sum_{k \neq i, j} v_{k}\left(a_{k}\right)-\sum_{k \neq i, j} v_{k}\left(b_{k}\right)=\delta \neq 0 .
$$

Furthermore, (1b) and (1c) imply

$$
\begin{aligned}
v_{i}\left(x_{i}^{\tau}\right)+v_{j}\left(x_{j}^{\tau}\right)+\sum_{k \neq i, j} v_{k}\left(a_{k}\right) & =0, \\
v_{i}\left(x_{i}^{\tau+1}\right)+v_{j}\left(x_{j}^{\tau}\right)+\sum_{k \neq i, j} v_{k}\left(b_{k}\right) & =0 .
\end{aligned}
$$

This implies that, for all $\tau \in \mathbb{T}$, we have

$$
v_{i}\left(x_{i}^{\tau+1}\right)-v_{i}\left(x_{i}^{\tau}\right)=\delta \neq 0 .
$$


Similarly, (1a) and (1b) imply

$$
\begin{aligned}
v_{i}\left(x_{i}^{\tau}\right)+v_{j}\left(x_{j}^{\tau-1}\right)+\sum_{k \neq i, j} v_{k}\left(b_{k}\right) & =0, \\
v_{i}\left(x_{i}^{\tau}\right)+v_{j}\left(x_{j}^{\tau}\right)+\sum_{k \neq i, j} v_{k}\left(a_{k}\right) & =0 .
\end{aligned}
$$

This implies that, for all $\tau \in \mathbb{T}$, we have

$$
v_{j}\left(x_{j}^{\tau-1}\right)-v_{i}\left(x_{i}^{\tau}\right)=\delta \neq 0 .
$$

Hence, the elements of a diagonal standard sequence are equally spaced in the numerical representation.

\section{Definition 6 (Strictly bounded diagonal standard sequence)}

Let $i, j \in N$ with $i \neq j$ and $\mathbb{T}$ be a set of consecutive integers. Let $\left\{x_{i}^{\tau} \in X_{i}\right.$, $\left.x_{j}^{\tau} \in X_{j},: \tau \in \mathbb{T}\right\}$, be a diagonal standard sequence on $i, j \in N$. We say that this diagonal standard sequence is strictly bounded on $i \in N$ if there are $\underline{x}_{i}, \bar{x}_{i} \in X_{i}$ such that $\bar{x}_{i} \succ_{i} x_{i}^{\tau}$ and $x_{i}^{\tau} \succ_{i} \underline{x}_{i}$ for all $\tau \in \mathbb{T}$.

Suppose that a diagonal standard sequence on $i, j \in N$ is strictly bounded on $i \in N$ by $\underline{x}_{i}$ and $\bar{x}_{i} \in X_{i}$. It is simple to check that we must have

$$
v_{i}\left(\underline{x}_{i}\right)<v_{i}\left(x_{i}^{\tau}\right)<v_{i}\left(\bar{x}_{i}\right)
$$

Because $v_{i}\left(x_{i}^{\tau+1}\right)-v_{i}\left(x_{i}^{\tau}\right)=\delta \neq 0$ the set $\mathbb{T}$ must be finite. This is the motivation for our Archimedean condition stated below that is clearly necessary for the existence of an additive representation.

\section{Definition 7 (Archimedean condition)}

Let $i, j \in N$ with $i \neq j$ and $\mathbb{T}$ be a set of consecutive integers. Let $\left\{x_{i}^{\tau} \in X_{i}\right.$, $\left.x_{j}^{\tau} \in X_{j},: \tau \in \mathbb{T}\right\}$, be a diagonal standard sequence on $i, j \in N$. If this standard sequence is strictly bounded on $i$ or on $j$ then the set $\mathbb{T}$ is finite.

Under the unrestricted solvability assumption stated below, it is simple to check that a diagonal standard sequence on $i, j \in N$ is strictly bounded on $i$ iff it is strictly bounded on $j$. As in classical conjoint measurement, the Archimedean axiom is not particularly intuitive. Its role is to forbid the existence of elements of $X_{i}$ that would be so good or so bad that a standard sequence would not be able to reach them. 


\subsection{Structural axioms}

Our main unnecessary assumption is a strong solvability assumption that says that category $\mathscr{F}$ can always be reached by modifying an evaluation on a single attribute. It cannot be overemphasized that this is a very strong hypothesis that will force all functions $v_{i}$ used in a representation in model $(A)$ to be unbounded.

\section{Definition 8 (Unrestricted solvability)}

We say that $\langle\mathscr{A}, \mathscr{F}, \mathscr{U}\rangle$ satisfies unrestricted solvability if, for all $i \in N$ and all $x_{-i} \in X_{-i},\left(x_{i}, x_{-i}\right) \in \mathscr{F}$, for some $x_{i} \in X_{i}$.

On top of unrestricted solvability, we will also suppose that $\langle\mathscr{A}, \mathscr{F}, \mathscr{U}\rangle$ is a nondegenerate partition. This is a rather mild restriction.

Let us note that if $\langle\mathscr{A}, \mathscr{F}, \mathscr{U}\rangle$ is a non-degenerate partition satisfying unrestricted solvability and that is strongly linear and strongly thin, then, for all $i \in N$, there are $z_{i}, w_{i} \in X_{i}$ such that $z_{i} \succ_{i} w_{i}$. Indeed using non-degeneracy, we know that $x \in \mathscr{A}$, for some $x \in X$. Using unrestricted solvability, we have $\left(y_{i}, x_{-i}\right) \in \mathscr{F}$, for some $y_{i} \in X_{i}$. This implies that $x_{i} \sim_{i} y_{i}$ is impossible. Therefore, under the above conditions, all attributes are influent for $\langle\mathscr{A}, \mathscr{F}, \mathscr{U}\rangle$.

\subsection{Results}

Our first result gives conditions ensuring the existence of an additive representation with tight uniqueness properties.

\section{Proposition 1}

Suppose that $\langle\mathscr{A}, \mathscr{F}, \mathscr{U}\rangle$ is an ordered partition of a set $X=X_{1} \times X_{2} \times \cdots \times X_{n}$ with $n \geq 3$. Suppose that $\langle\mathscr{A}, \mathscr{F}, \mathscr{U}\rangle$ is non-degenerate and satisfies unrestricted solvability, strong linearity, strong thinness and the Archimedean condition. If $n=3$, suppose furthermore that $\langle\mathscr{A}, \mathscr{F}, \mathscr{U}\rangle$ satisfies the Thomsen condition. Then there is an additive representation $\left\langle v_{i}\right\rangle_{i \in N}$ of $\langle\mathscr{A}, \mathscr{F}, \mathscr{U}\rangle$ in model $(A)$.

Under the above conditions, $\left\langle u_{i}\right\rangle_{i \in N}$ and $\left\langle v_{i}\right\rangle_{i \in N}$ are two additive representations of $\langle\mathscr{A}, \mathscr{F}, \mathscr{U}\rangle$ in model $(A)$ iff there are real numbers $\beta_{1}, \beta_{2}, \ldots, \beta_{n}, \alpha$ with $\alpha>0$ and $\sum_{i=1}^{n} \beta_{i}=0$ such that for all $i \in N$ and all $x_{i} \in X_{i}, v_{i}\left(x_{i}\right)=\alpha u_{i}\left(x_{i}\right)+\beta_{i}$.

The proof appears in section A in the supplementary material to this paper.

Let us give two typical examples of ordered partitions satisfying the conditions of Proposition 1. The first corresponds to discrete equally spaced structure. The second is a continuous structure.

\section{Example 1}

Let $X=\mathbb{Z}^{3}$ with $\mathscr{A}=\left\{x \in X: x_{1}+x_{2}+x_{3}>0\right\}$ and $\mathscr{F}=\left\{x \in X: x_{1}+x_{2}+x_{3}=\right.$ $0\}$. Taking, for all $i \in N, v_{i}\left(x_{i}\right)=x_{i}$ clearly gives a representation of this ordered partition in model $(A)$. 


\section{Example 2}

Let $X=\mathbb{R}^{3}$ with $\mathscr{A}=\left\{x \in X: x_{1}+x_{2}+x_{3}>0\right\}$ and $\mathscr{F}=\left\{x \in X: x_{1}+x_{2}+x_{3}=\right.$ $0\}$. Taking, for all $i \in N, v_{i}\left(x_{i}\right)=x_{i}$ clearly gives a representation of this ordered partition in model $(A)$.

Observe that for the uniqueness part of the above proposition, we have supposed that the threshold 0 was fixed. This may give the impression that this uniqueness result is stronger than what it really is. If the threshold used for $\mathscr{F}$ is taken to be variable from one representation to another, it is easy to see that one goes from an additive representation to another one simply by multiplying all functions $u_{i}$ by the same positive constant and adding a constant $\beta_{i}$ to each of them. If the first representation uses a null threshold, the second one will use a threshold equal to $\sum_{i=1}^{n} \beta_{i}$.

Proposition 1 uses strong linearity and strong thinness. Although this allows to simply grasp the conditions underlying the result, this involves some redundancy. For instance, it is clear that conditions $\mathscr{A}$-linear $_{I}$ and $\mathscr{A}$-linear $-I$ are equivalent. This brings us to the main results in this section that show how to sharpen the conditions used above. The uniqueness of the representation obtained with these two results is the same as in Proposition 1.

We say that $\langle\mathscr{A}, \mathscr{F}, \mathscr{U}\rangle$ is $\nu$-A्A-linear if it satisfies $\mathscr{A}$-linear $_{I}$ for all nonempty proper subset $I$ of $N$ such that $|I|=\nu>0$. We use a similar convention for $\nu$ - $\mathscr{F}$-linear, $\nu$ - $\mathscr{A} \mathscr{F}$-linear, $\nu$-linear and $\nu$-thin.

\section{Theorem 1}

Let $\langle\mathscr{A}, \mathscr{F}, \mathscr{U}\rangle$ be an ordered partition of a set $X=X_{1} \times X_{2} \times X_{3}$. If $\langle\mathscr{A}, \mathscr{F}, \mathscr{U}\rangle$ is non-degenerate and satisfies unrestricted solvability, the Archimedean condition, 1$\mathscr{A}$-linear, 1-F्F-linear, 1-thin and the Thomsen condition, then there is an additive representation of $\langle\mathscr{A}, \mathscr{F}, \mathscr{U}\rangle$.

\section{Theorem 2}

Suppose that $\langle\mathscr{A}, \mathscr{F}, \mathscr{U}\rangle$ is a non-degenerate ordered partition of a set $X=X_{1} \times$ $X_{2} \times \cdots \times X_{n}$ with $n \geq 4$. Suppose that $\langle\mathscr{A}, \mathscr{F}, \mathscr{U}\rangle$ satisfies unrestricted solvabili-

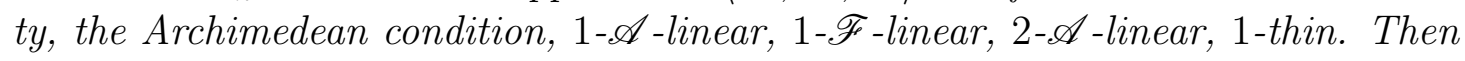
there is an additive representation of $\langle\mathscr{A}, \mathscr{F}, \mathscr{U}\rangle$.

Theorems 1 and 2 are proved in section B in the supplementary material to this paper. They give reasonably simple conditions ensuring the existence of additive representations with tight uniqueness properties. In Section 6 , we relate them to the existing literature on the subject and discuss their interest. 


\section{$5 \quad$ Beyond threefold partitions}

The approach taken above can be generalized to cover the case of more than two categories separated by a single frontier. This case is dealt with separately since it will use our results for the case of two categories separated by a single frontier as a lemma.

\subsection{The setting}

Let $r \geq 2$ be an integer. Let $R=\{1,2, \ldots, r\}$. An $r$-fold ordered covering of the set $X$ is a collection of nonempty sets $\left\langle C^{1}, C^{2}, \ldots, C^{r}\right\rangle$ such that $C^{1} \cup C^{2} \cup \cdots \cup C^{r}=X$ and $C^{k} \cap C^{\ell}=\varnothing$, for all $k, \ell \in R$ such that $|k-\ell|>1$. We define for all $k \in R$, $C_{\geq}^{k}=\bigcup_{j=k}^{r} C^{j}, C_{\leq}^{k}=\bigcup_{j=1}^{k} C^{j}$. Note that we use here superscripts for categories and not to indicate exponentiation.

The alternatives in $C^{k} \cap C^{k+1}$ are interpreted as lying at the frontier between categories $C^{k}$ and $C^{k+1}$. For $k \in R$, we define $\Delta^{k}=C^{k} \backslash\left[C^{k-1} \cup C^{k+1}\right]$, with the convention that $C^{0}=C^{r+1}=\varnothing$. The alternatives in $\Delta^{k}$ are therefore the alternatives that belong to $C^{k}$ and do not lie at the frontier between $C^{k}$ and one of its adjacent categories. We define, for all $k \in\{1,2, \ldots, r-1\}, \Delta_{\geq}^{k}=\Delta^{k} \cup C_{\geq}^{k+1}$ and $\Delta_{\leq}^{k}=\Delta^{k} \cup C_{\leq}^{k-1}$.

The ordered covering $\left\langle C^{1}, C^{2}, \ldots, C^{r}\right\rangle$ may therefore be viewed as an ordered partition $\left\langle\Delta^{1}, C^{1} \cap C^{2}, \Delta^{2}, C^{2} \cap C^{3}, \Delta^{3}, \ldots, C^{r-1} \cap C^{r}, \Delta^{r}\right\rangle$. Clearly, for all $k \in$ $\{1,2, \ldots, r-1\}$, this ordered partition generates a threefold ordered partition $\left\langle\Delta_{\leq}^{k}, C^{k} \cap C^{k+1}, \Delta_{\geq}^{k+1}\right\rangle$. Each of these threefold partitions can be analyzed using the results in Section 4.

We say that $\left\langle C^{1}, C^{2}, \ldots, C^{r}\right\rangle$ is non-degenerate if $\Delta^{k} \neq \varnothing$, for all $k \in R$.

\subsection{The model}

We consider an ordered covering $\left\langle C^{k}\right\rangle_{k \in R}$, possibly with $r \geq 3$. We are interested in a representation of $\left\langle C^{k}\right\rangle_{k \in R}$ such that, for all $x \in X$, and all $k \in R$,

$$
x \in C^{k} \Leftrightarrow \sigma^{k-1} \leq \sum_{i=1}^{n} v_{i}\left(x_{i}\right) \leq \sigma^{k},
$$

with the convention that $\sigma^{0}=-\infty, \sigma^{r}=+\infty$ and where $\sigma^{1}, \sigma^{2}, \ldots, \sigma^{r-1}$ are real numbers such that $\sigma^{1}<\sigma^{2}<\cdots<\sigma^{r-1}$ and $v_{i}$ is a real-valued function on $X_{i}$. This clearly implies that $x \in C^{k} \cap C^{k+1} \Leftrightarrow \sum_{i=1}^{n} v_{i}\left(x_{i}\right)=\sigma^{k}$ and $x \in \Delta_{\geq}^{k+1} \Leftrightarrow$ $\sum_{i=1}^{n} v_{i}\left(x_{i}\right)>\sigma^{k}$. Hence, when model $\left(A^{*}\right)$ holds, each of the threefold partitions (for $r \in\{1,2, \ldots, r-1\}$ ) $\left\langle\Delta_{\leq}^{k}, C^{k} \cap C^{k+1}, \Delta_{>}^{k+1}\right\rangle$ have a representation in model $(A)$ and the basis of our analysis will be the results obtained for model $(A)$ in Section 4 . 


\subsection{Axioms and result}

Our strategy is as follows. We will suppose that one of the threefold partition $\left\langle\Delta_{\leq}^{k}, C^{k} \cap C^{k+1}, \Delta_{\geq}^{k+1}\right\rangle$ satisfies the conditions of Theorem 1 (when $n=3$ ) or of Theorem 2 (when $n \geq 4$ ). We will then add extra conditions ensuring that the additive representation of $\left\langle\Delta_{<}^{k}, C^{k} \cap C^{k+1}, \Delta_{>}^{k+1}\right\rangle$ also gives a representation of $\left\langle C^{k}\right\rangle_{k \in R}$ in model $\left(A^{*}\right)$.

Our first additional condition strengthens thinness so that if two alternatives differing in only one attribute are caught in a frontier, changing the $n-1$ common values of these two alternatives will never allow to distinguish them.

\section{Definition 9 (Generalized 1-thinness)}

We say that $\left\langle C^{k}\right\rangle_{k \in R}$ satisfies generalized 1-thinness if for all $h \in\{1,2, \ldots, r-1\}$, all $i \in N$, all $a_{i}, b_{i} \in X_{i}$ and all $c_{-i}, d_{-i} \in X_{-i},\left[\left(a_{i}, c_{-i}\right) \in C^{h} \cap C^{h+1}\right.$ and $\left.\left(b_{i}, c_{-i}\right) \in C^{h} \cap C^{h+1}\right] \Rightarrow\left[\left(a_{i}, d_{-i}\right) \in C^{k} \Leftrightarrow\left(b_{i}, d_{-i}\right) \in C^{k}\right.$, for all $\left.k \in R\right]$.

It is easy to see that generalized 1-thinness is necessary for model $\left(A^{*}\right)$, since $\left[\left(a_{i}, c_{-i}\right) \in C^{h} \cap C^{h+1}\right.$ and $\left.\left(b_{i}, c_{-i}\right) \in C^{h} \cap C^{h+1}\right]$ imply $v_{i}\left(a_{i}\right)=v_{i}\left(b_{i}\right)$. This condition clearly implies that all the threefold partitions $\left\langle\Delta_{\leq}^{k}, C^{k} \cap C^{k+1}, \Delta_{\geq}^{k+1}\right\rangle$ induced by $\left\langle C^{k}\right\rangle_{k \in R}$ are 1-thin.

Our second additional condition aims at imposing that two distinct frontiers between categories have the same shape. It says that when the tradeoff between $a_{i}$ and $a_{j}$ is "equivalent" to the tradeoff between $b_{i}$ and $b_{j}$ because both are caught in the same frontier when adjoined a common element in $X_{-i j}$, then a similar conclusion will hold when considering other frontiers.

\section{Definition 10 (Parallelism)}

An ordered covering $\left\langle C^{k}\right\rangle_{k \in R}$ satisfies parallelism if

$$
\left.\begin{array}{rl}
\left(a_{i}, a_{j}, c_{-i j}\right) & \in C^{h} \cap C^{h+1} \\
\left(b_{i}, b_{j}, c_{-i j}\right) & \in C^{h} \cap C^{h+1} \\
\left(a_{i}, a_{j}, d_{-i j}\right) & \in C^{k} \cap C^{k+1}
\end{array}\right\} \Rightarrow\left(b_{i}, b_{j}, d_{-i j}\right) \in C^{k} \cap C^{k+1} .
$$

for all $h, k \in\{1,2, \ldots, r-1\}$, all $a_{i}, b_{i} \in X_{i}$, all $a_{j}, b_{j} \in X_{j}$ and all $c_{-i j}, d_{-i j} \in$ $X_{-i j}$.

It is easy to check that parallelism is a necessary condition for model $\left(A^{*}\right)$. Indeed, $\left(a_{i}, a_{j}, c_{-i j}\right) \in C^{h} \cap C^{h+1}$ and $\left(b_{i}, b_{j}, c_{-i j}\right) \in C^{h} \cap C^{h+1}$ imply in model $\left(A^{*}\right)$ that $v_{i}\left(a_{i}\right)+v_{j}\left(a_{j}\right)=v_{i}\left(b_{i}\right)+v_{j}\left(b_{j}\right)$. Hence if $\left(a_{i}, a_{j}, d_{-i j}\right) \in C^{k} \cap C^{k+1}$, i.e., $v_{i}\left(a_{i}\right)+$ $v_{j}\left(a_{j}\right)+\sum_{k \neq i, j} v_{k}\left(d_{k}\right)=\sigma^{k}$, we have $v_{i}\left(b_{i}\right)+v_{j}\left(b_{j}\right)+\sum_{k \neq i, j} v_{k}\left(d_{k}\right)=\sigma^{k}$, so that $\left(b_{i}, b_{j}, d_{-i j}\right) \in C^{k} \cap C^{k+1}$.

The next two conditions ensures a minimal consistency between all the twofold coverings that are induced from $\left\langle C^{k}\right\rangle_{k \in R}$. 


\section{Definition 11 ( $\Upsilon$-Mixed-1-linearity)}

We say that $\left\langle C^{k}\right\rangle_{k \in R}$ satisfies mixed-1-linearity w.r.t. the frontiers or, for short, $\Upsilon$-mixed-1-linearity, if

$$
\left.\begin{array}{c}
\left(x_{i}, a_{-i}\right) \in C^{k-1} \cap C^{k} \\
\text { and } \\
\left(y_{i}, b_{-i}\right) \in C^{h-1} \cap C^{h}
\end{array}\right\} \Rightarrow\left\{\begin{array}{c}
\left(y_{i}, a_{-i}\right) \in C_{\geq}^{k} \\
\text { or } \\
\left(x_{i}, b_{-i}\right) \in C_{\geq}^{h}
\end{array}\right.
$$

for all $k, \ell \in R$, all $i \in N$, all $x_{i}, y_{i} \in X_{i}$ and all $a_{-i j}, b_{-i j} \in X_{-i j}$.

\section{Definition 12 ( $\Delta$-Mixed-1-linearity)}

We say that $\left\langle C^{k}\right\rangle_{k \in R}$ satisfies mixed-1-linearity with respect to the interior or, for short, $\Delta$-mixed-1-linearity if

$$
\left.\begin{array}{c}
\left(x_{i}, a_{-i}\right) \in \Delta_{\geq}^{k} \\
\text { and } \\
\left(y_{i}, b_{-i}\right) \in \Delta_{\geq}^{h}
\end{array}\right\} \Rightarrow\left\{\begin{array}{c}
\left(y_{i}, a_{-i}\right) \in \Delta_{\geq}^{k} \\
\text { or } \\
\left(x_{i}, b_{-i}\right) \in \Delta_{\geq}^{h}
\end{array}\right.
$$

for all $k, \ell \in R$, all $i \in N$, all $x_{i}, y_{i} \in X_{i}$ and all $a_{-i j}, b_{-i j} \in X_{-i j}$.

These two conditions are clearly necessary for model $\left(A^{*}\right)$. For instance, if $\left(x_{i}, a_{-i}\right) \in$ $C^{k-1} \cap C^{k}$ and $\left(y_{i}, b_{-i}\right) \in C^{h-1} \cap C^{h}$, we have $v_{i}\left(x_{i}\right)+\sum_{j \neq i} v_{j}\left(a_{j}\right)=\sigma^{k-1}$ and $v_{i}\left(y_{i}\right)+\sum_{j \neq i} v_{j}\left(b_{j}\right)=\sigma^{h-1}$. If $v_{i}\left(x_{i}\right) \geq v_{i}\left(y_{i}\right)$, it follows that $v_{i}\left(x_{i}\right)+\sum_{j \neq i} v_{j}\left(b_{j}\right) \geq$ $\sigma^{h-1}$, so that $\left(x_{i}, b_{-i}\right) \in C_{\geq}^{h}$. Otherwise, we have $v_{i}\left(y_{i}\right)>v_{i}\left(x_{i}\right)$, which implies $v_{i}\left(y_{i}\right)+\sum_{j \neq i} v_{j}\left(a_{j}\right)>\sigma^{k-1}$, so that $\left(y_{i}, a_{-i}\right) \in C_{\geq}^{k}$. In Bouyssou and Marchant (2008a), we show the importance of generalized 1-thinness $\Upsilon$-Mixed-1-linearity and $\Delta$-Mixed-1-linearity to obtain a strictly increasing decomposable representation of $\left\langle C^{k}\right\rangle_{k \in R}$, i.e, a model that is similar to $\left(A^{*}\right)$ except that the sum has been replaced by a function $F$ that is strictly increasing in all its arguments.

Observe that if $\left\langle C^{k}\right\rangle_{k \in R}$ satisfies $\Upsilon$-mixed-1-linearity and $\Delta$-mixed-1-linearity

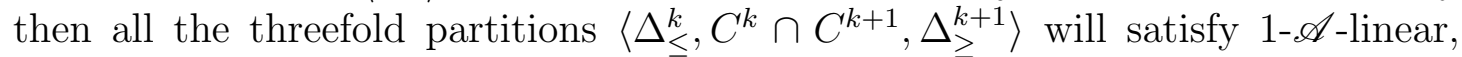
1- $\mathscr{F}$-linear, with the convention that $\mathscr{A}=\Delta_{\geq}^{k+1}$ and $\mathscr{F}=C^{k} \cap C^{k+1}$.

Our final additional condition is a strengthening of unrestricted solvability saying that starting with any alternative one can reach any frontier by modifying this alternative on a single attribute. This is a strong condition that is clearly not necessary for model $\left(A^{*}\right)$.

Definition 13 (Unrestricted solvability w.r.t. all frontiers)

An ordered covering $\left\langle C^{k}\right\rangle_{k \in R}$ satisfies unrestricted solvability w.r.t. all frontiers if if, for all $h \in\{1,2, \ldots, r-1\}$, all $i \in N$ and all $a_{-i} \in X_{-i},\left(a_{i}, a_{-i}\right) \in C^{h} \cap C^{h+1}$, for some $a_{i} \in X_{i}$.

Our main result in this section is the following: 


\section{Theorem 3}

Let $\left\langle C^{k}\right\rangle_{k \in R}$ be a non-degenerate ordered covering of $X$ such that:

1. $\left\langle C^{k}\right\rangle_{k \in R}$ satisfies generalized 1-thinness, $\Upsilon$-mixed-1-linearity, $\Delta$-Mixed-1-linearity, parallelism and unrestricted solvability w.r.t. all frontiers,

2. for some $k \in\{1,2, \ldots, r-1\}$, the threefold partition $\left\langle\Delta_{\leq}^{k}, C^{k} \cap C^{k+1}, \Delta_{\geq}^{k+1}\right\rangle$ satisfies the Archimedean condition and either Thomsen condition (if $n=3$ ) or or 2 - $\mathscr{A}$-linear (if $n \geq 4$ ) (using the obvious convention that $\mathscr{A}=\Delta_{\geq}^{k+1}$ ).

Then, there is an additive representation of $\left\langle C^{k}\right\rangle_{k \in R}$ in model $\left(A^{*}\right)$.

Under the above conditions, $\left\langle u_{i}\right\rangle_{i \in N}$ and $\left\langle v_{i}\right\rangle_{i \in N}$ are two additive representations of $\left\langle C^{k}\right\rangle_{k \in R}$ using the same thresholds $\sigma^{1}<\sigma^{2}<\cdots<\sigma^{r-1}$ iff there are real numbers $\beta_{1}, \beta_{2}, \ldots, \beta_{n}$ with $\sum_{i=1}^{n} \beta_{i}=0$ such that for all $i \in N$ and all $x_{i} \in X_{i}$, $v_{i}\left(x_{i}\right)=u_{i}\left(x_{i}\right)+\beta_{i}$.

The proof appears in section $\mathrm{C}$ in the supplementary material to this paper.

The following section discusses our results and positions them w.r.t. the existing literature on the subject.

\section{Relation to the literature and discussion}

\subsection{Relation to the literature}

This paper has shown how to adapt classical results of conjoint measurement giving conditions guaranteeing the existence of additive representations of binary relations to the case of ordered partitions.

The only results we are aware of that deal with a problem comparable to ours are the ones in Vind (1991) (almost identical results appear in Vind, 2003, Ch. 5 $\& 9$ ). Vind (1991) considers a subset $\mathscr{A}$ of a product set $X$ with $n \geq 4$. Condition $\mathscr{A}$-linear ${ }_{I}$ is required to hold for all nonempty proper subset $I$ of $N$. This allows to define a weak order on each $X_{i}$ in an obvious way (i.e., $x_{i} \succsim_{i}^{\mathscr{A}} y_{i} \Leftrightarrow$ [for all $a_{-i} \in$ $\left.\left.X_{-i},\left(y_{i}, a_{-i}\right) \in \mathscr{A} \Rightarrow\left(x_{i}, a_{-i}\right) \in \mathscr{A}\right]\right)$. Using this weak order, Vind imposes that each set $X_{i}$ is connected in the order topology generated by $\succsim_{i}^{\mathscr{A}}$. The product set $X$ is endowed with the product topology. All attributes are required to be essential in the following sense. For all $i \in N$ and for all $x_{i} \in X_{i}$, there are $a_{-i}, b_{-i} \in X_{-i}$ such that $\left(x_{i}, a_{-i}\right) \in \mathscr{A}$ and $\left(x_{i}, b_{-i}\right) \notin \mathscr{A}$.

The main result in Vind (1991) is as follows.

Theorem (Vind, 1991, Th. 1, p. 122)

Let $X$ be such that $n \geq 4$ and, for all $i \in N,\left|X_{i}\right|>1$. Suppose that:

1. all attributes are essential, 
2. for all nonempty proper subset I of $N$, condition $\mathscr{A}$-linear I holds,

3. for all $i \in N, X_{i}$ is connected in the order topology induced by the weak order $\succsim_{i}^{\mathscr{A}}$, and

4. $\mathscr{A}$ is open in the product topology on $X$,

then there are real-valued $v_{i}$ on $X_{i}$ such that, for all $x \in X, x \in \mathscr{A} \Leftrightarrow \sum_{i=1}^{n} v_{i}\left(x_{i}\right)>$ 0 . Under the above conditions, the uniqueness of the functions $v_{i}$ is as in Theorem 1.

Compared to the above result, our own results have advantages and disadvantages.

Let us begin by the positive side. We have dealt with the case of three attributes and the case with more than two ordered categories separated by a single frontier. These two cases are not dealt with in Vind (1991). We have also outlined an assessment protocol of the functions $v_{i}$, a point that is not explicitly tackled in Vind (1991). Moreover, our results do not exclude the case of discrete equally spaced structures, such as the one in Example 1, a case that is not covered by Vind's (1991) result since it clearly violates the connectedness of each $X_{i}$. A final argument on the positive side is the simplicity of our results that are rather direct extensions of classical results. Vind's (1991) proofs are based on theory of mean groupoïds. Besides the fact that his proofs are somewhat sketchy, we feel that ours are far simpler.

On the negative side, we have to mention two main points. First, as already stressed, the simplicity of our results are mainly due to our use of a strong solvability assumption. Indeed, unrestricted solvability forces all functions $v_{i}$ used in a representation in model $(A)$ to be unbounded. This not the case in Vind (1991). It is important to stress that the approach taken here vitally depends on this hypothesis. We investigate in Bouyssou and Marchant (2009) another approach that uses the results in Chateauneuf and Wakker (1993) allowing to build additive representations of weak orders defined on subsets of product sets (since this approach appeals to connectedness, it does not allow to cover the case of discrete equally spaced structures as in Example 1 however). Another weak point of our results compared to Vind's (1991) is that we use conditions that explicitly appeal to the frontier $\mathscr{F}$. In the topological setting of Vind (1991), the treatment of the frontier may remain implicit: since $\mathscr{A}$ is open, the element of $\mathscr{F}$ may be viewed as the elements belonging to $\operatorname{cl}(\mathscr{A}) \backslash \mathscr{A}$, where 'cl' denotes the closure operator. In our algebraic setting, we need to impose conditions dealing with the alternatives in $\mathscr{F}$. Clearly, such conditions are less intuitive than conditions that would only involve $\mathscr{A}$ and $\mathscr{U}$. In an algebraic setting, this seems unavoidable however. 


\subsection{Discussion}

The analysis in this paper was conceived within the framework of conjoint measurement: the only primitive in our model is a judgment on the objects in the product set $X$. Such a framework is especially attractive for psychologists. Indeed, they often have to test whether data collected in experiments are or not compatible with a given representation model. They also commonly want to design and conduct experiments that would try to falsify the hypotheses underlying a given model. Our results are partly intended to facilitate such analyses. They underline the importance of a small number of conditions (mainly linearity and thinness) for the existence of additive representations of ordered partitions. These conditions are reasonably simple and could well be the subject of experimental research. This is however clearly outside the scope of this paper.

Most readers of this journal will not be psychologists however. Hence, it may be important to explain why our results may be of interest to people working in the field of MCDM.

Most MCDM techniques using an additive value function model for sorting, such as UTADIS (see Jacquet-Lagrèze, 1995, Zopounidis and Doumpos, 2000b), proceed as follows. The DM is asked to assign some alternatives in $X$ to ordered categories. A Linear Programming (LP) model is then used to test whether or not the collected information is compatible with an additive model. If there is such a model, the method will then try to propose an assignment of all alternatives in $X$ to ordered categories. Note that the elaboration of the prescription should clearly deal with the indetermination of the value function obtained as the result of the optimization model. Indeed, in general, there will be infinitely many additive models (using the same normalization) that allow to recover the information given by the DM. If there is no additive model compatible with the information that was collected, one may try to use an additive model that is as "close" as possible to this information. Alternatively, one may try to interact with the DM explaining why the information is incompatible with an additive model and suggesting the revision of some of the initial judgments. This seems to be quite different from the analysis in this paper. Therefore one could ask whether or not our results can be useful for the practitioner of MCDM. We view our contribution for MCDM as twofold.

First, we have shown that our results give clues on the construction of a structured assessment protocol of additive value functions for sorting. This gives a direct way to assess an additive value function with tight uniqueness properties, contrary to what is obtained with LP-based models. A second possible virtue of our results is linked to the use of the axiomatic method as a tool to understand and compare models. Since various sorting models have now been axiomatized (see Bouyssou and Marchant, 2007a,b, Goldstein, 1991, Słowiński et al., 2002), 
our results can be used as a basis of comparison of different sorting techniques. For instance, Bouyssou and Marchant (2007a) have shown that ELECTRE TRI also appeals to an idea of linearity on each attribute, which shows that ELECTRE TRI and the additive value function model $d o$ have some common features. The main distinctive feature of ELECTRE TRI is that it induces on each attribute a weak order that is coarse (i.e., distinguishing only very few distinct equivalence classes). In the additive value function model, linearity is extended to subsets of attributes and the resulting weak orders have a much richer structure. Our results may be viewed as a further contribution towards a systematic axiomatic analysis of ordered partitions in a conjoint measurement framework. We are confident that such an analysis will not only deepen our understanding of MCDM techniques for sorting but might also give rise to new methods and assessment protocols. 


\section{Supplementary material}

\section{A Proof of Proposition 1}

\section{A.1 Preliminary results}

Take any $j \in N$. Define on the set $\prod_{i \neq j} X_{j}$ the binary relation $\succsim^{(j)}$ letting, for all $x_{-j}, y_{-j} \in X_{-j}$

$$
x_{-j} \succsim^{(j)} y_{-j} \Leftrightarrow\left(a_{j}, x_{-j}\right) \in \mathscr{A} \mathscr{F} \text { and }\left(a_{j}, y_{-j}\right) \in \mathscr{F} \mathscr{U}
$$

for some $a_{j} \in X_{j}$. We use $\succ^{(j)}$ and $\sim^{(j)}$ as is usual. Our proof rests on the following lemma showing that under the conditions of Proposition 1 , the relation $\succsim^{(j)}$ will satisfy the classical conditions ensuring the existence of an additive representation for this relation.

\section{Lemma A-1}

Let $\langle\mathscr{A}, \mathscr{F}, \mathscr{U}\rangle$ be an ordered covering on a set $X$. Suppose that this covering is strongly linear and strongly thin. Suppose furthermore that unrestricted solvability holds. Let $j \in N$. We have:

1. For all $x_{-j}, y_{-j} \in X_{-j}$,

$$
x_{-j} \succsim^{(j)} y_{-j} \Leftrightarrow x_{-j} \succsim_{-j} y_{-j},
$$

so that $\succsim^{(j)}$ is complete and transitive.

2. For all $x_{-j}, y_{-j} \in X_{-j}$,

$$
x_{-j} \sim^{(j)} y_{-j} \Leftrightarrow\left\{\begin{array}{l}
\left(a_{j}, x_{-j}\right) \in \mathscr{F} \\
\left(a_{j}, y_{-j}\right) \in \mathscr{F}
\end{array}\right\} \text { for some } a_{j} \in X_{j} .
$$

3. For all $x_{-j}, y_{-j} \in X_{-j}$,

$$
x_{-j} \succ^{(j)} y_{-j} \Leftrightarrow\left\{\begin{array}{l}
\left(a_{j}, x_{-j}\right) \in \mathscr{A} \\
\left(a_{j}, y_{-j}\right) \in \mathscr{F}
\end{array}\right\} \text { for some } a_{j} \in X_{j} .
$$

4. The binary relation $\succsim^{(j)}$ is independent, i.e., for all $i \in N \backslash\{j\}$, all $x_{i}, y_{i} \in X_{i}$ and all $a_{-i j}, b_{-i j} \in X_{-i j}$,

$$
\left(x_{i}, a_{-i j}\right) \succsim^{(j)}\left(x_{i}, b_{-i j}\right) \Leftrightarrow\left(y_{i}, a_{-i j}\right) \succsim^{(j)}\left(y_{i}, b_{-i j}\right) .
$$


5. The binary relation $\succsim^{(j)}$ satisfies unrestricted solvability, i.e., for all $y_{-j} \in$ $X_{-j}$, all $i \in N \backslash\{j\}$ and all $a_{-i j} \in X_{-i j},\left(x_{i}, a_{-i j}\right) \sim^{(j)} y_{-j}$, for some $x_{i} \in X_{i}$.

6. If $\langle\mathscr{A}, \mathscr{F}, \mathscr{U}\rangle$ is non-degenerate, there is at least one essential attribute for $\succsim^{(j)}$, i.e., $\left(x_{i}, a_{-i j}\right) \succ^{(j)}\left(y_{i}, a_{-i j}\right)$, for some $i \in N \backslash\{j\}$, some $x_{i}, y_{i} \in X_{i}$ and some $a_{-i j} \in X_{-i j}$.

7. If $\langle\mathscr{A}, \mathscr{F}, \mathscr{U}\rangle$ satisfies the Archimedean condition, then $\succsim^{(j)}$ satisfies the Archimedean condition. More precisely, let $\mathbb{T}$ be any set of consecutive integers (positive or negative, finite or infinite). We say that the set $\left\{x_{i}^{\tau} \in\right.$ $\left.X_{i}: \tau \in \mathbb{T}\right\}$ is a standard sequence for $\succsim^{(j)}$ on attribute $i \in N$ if there are $a_{-i j}, b_{-i j} \in X_{-i j}$ such that $\left(y_{i}, a_{-i j}\right) \succ^{(j)}\left(y_{i}, b_{-i j}\right)$, for some $y_{i} \in X_{i}$ and $\left(x_{i}^{\tau}, a_{-i j}\right) \sim^{(j)}\left(x_{i}^{\tau+1}, b_{-i j}\right)$, for all $\tau \in \mathbb{T}$. This standard sequence is said to be strictly bounded if there are $\underline{x}_{i}, \bar{x}_{i} \in X_{i}$ such that, for all $\tau \in \mathbb{T}$, $\left(\bar{x}_{i}, c_{-i j}\right) \succ^{(j)}\left(x_{i}^{\tau}, c_{-i j}\right) \succ^{(j)}\left(\underline{x}_{i}, c_{-i j}\right)$ for all $c_{-i j} \in X_{-i j}$. The relation $\succsim^{(j)}$ is said to satisfy the Archimedean condition, if, for all $i \in N \backslash\{j\}$, any standard sequence on attribute $i$ that is strictly bounded is finite.

8. Suppose that $n=3$ and let $N=\{i, j, k\}$. If $\langle\mathscr{A}, \mathscr{F}, \mathscr{U}\rangle$ satisfies the Thomsen condition, then $\succsim^{(j)}$ satisfies the Thomsen condition, i.e., for all $i, k \in N \backslash\{j\}$ with $i \neq k$, all $x_{i}, y_{i}, z_{i} \in X_{i}$ and all $x_{k}, y_{k}, z_{k} \in X_{k}$,

$$
\left.\begin{array}{c}
\left(x_{i}, x_{k}\right) \sim^{(j)}\left(y_{i}, y_{k}\right) \\
\text { and } \\
\left(y_{i}, z_{k}\right) \sim^{(j)}\left(z_{i}, x_{k}\right)
\end{array}\right\} \Rightarrow\left(x_{i}, z_{k}\right) \sim^{(j)}\left(z_{i}, y_{k}\right) .
$$

9. If there is an additive representation for $\succsim^{(j)}$, then there is an additive representation for $\langle\mathscr{A}, \mathscr{F}, \mathscr{U}\rangle$.

PROOF

Part 1. Suppose that $x_{-j} \succsim_{-j} y_{-j}$, so that, for all $a_{j} \in X_{j},\left(a_{j}, y_{-j}\right) \in \mathscr{A} \Rightarrow$ $\left(a_{j}, x_{-j}\right) \in \mathscr{A}$ and $\left(a_{j}, y_{-j}\right) \in \mathscr{F} \Rightarrow\left(a_{j}, x_{-j}\right) \in \mathscr{A} \mathscr{F}$. Using unrestricted solvability, we know that $\left(b_{j}, y_{-j}\right) \in \mathscr{F}$, for some $b_{j} \in X_{j}$. Because $x_{-j} \succsim_{-j} y_{-j}$, this implies that $\left(b_{j}, x_{-j}\right) \in \mathscr{A} \mathscr{F}$, so that $x_{-j} \succsim^{(j)} y_{-j}$.

Suppose now that $x_{-j} \succsim^{(j)} y_{-j}$ so that $\left(a_{j}, x_{-j}\right) \in \mathscr{A} \mathscr{F}$ and $\left(a_{j}, y_{-j}\right) \in \mathscr{F} \mathscr{U}$, for some $a_{j} \in X_{j}$. Suppose that $\operatorname{Not}\left[x_{-j} \succsim_{-j} y_{-j}\right]$. Using $(n-1)$-linear, we know that $\succsim_{-j}$ is complete so that we have $y_{-j} \succ_{-j} x_{-j}$. Using $(n-1)$-linear and $(n-1)$-thin, $y_{-j} \succ_{-j} x_{-j}$ and $\left(a_{j}, x_{-j}\right) \in \mathscr{A} \mathscr{F}$ imply $\left(a_{j}, y_{-j}\right) \in \mathscr{A}$, a contradiction.

Part 2. The $\Leftarrow$ part follows from the definition of $\succsim^{(j)}$. Let us prove the $\Rightarrow$ part. Suppose that $x_{-j} \sim^{(j)} y_{-j}$, so that for some $a_{j}, b_{j} \in X_{j}$,

$$
\begin{aligned}
\left(a_{j}, x_{-j}\right) & \in \mathscr{A F} \text { and }\left(a_{j}, y_{-j}\right) \in \mathscr{F} \mathscr{U}, \\
\left(b_{j}, y_{-j}\right) & \in \mathscr{A F} \text { and }\left(b_{j}, x_{-j}\right) \in \mathscr{F} \mathscr{U} .
\end{aligned}
$$


Using unrestricted solvability on attribute $j$, we know that there is a $c_{j} \in X_{j}$ such that $\left(c_{j}, x_{-j}\right) \in \mathscr{F}$. If $\left(c_{j}, y_{-j}\right) \in \mathscr{F}$, there is nothing to prove. Suppose that $\left(c_{j}, y_{-j}\right) \in \mathscr{U}$. Using $(n-1)$-linear, this implies that $x_{-j} \succ_{-j} y_{-j}$. Using $(n-1)$-linear and $(n-1)$-thin, $\left(b_{j}, y_{-j}\right) \in \mathscr{A} \mathscr{F}$ and $x_{-j} \succ_{-j} y_{-j}$ imply $\left(b_{j}, x_{-j}\right) \in$ $\mathscr{A}$, a contradiction. Similarly if $\left(c_{j}, y_{-j}\right) \in \mathscr{A}$, we obtain $y_{-j} \succ_{-j} x_{-j}$ so that $\left(a_{j}, x_{-j}\right) \in \mathscr{A} \mathscr{F}$ implies $\left(a_{j}, y_{-j}\right) \in \mathscr{A}$, a contradiction.

Part 3. Suppose that $x_{-j} \succ^{(j)} y_{-j}$. Using unrestricted solvability on attribute $j$, we know that $\left(a_{j}, y_{-j}\right) \in \mathscr{F}$, for some $a_{j} \in X_{j}$. We have either $\left(a_{j}, x_{-j}\right) \in \mathscr{A}$ or $\left(a_{j}, x_{-j}\right) \in \mathscr{F} \mathscr{U}$. The latter case implies $y_{-j} \succsim^{(j)} x_{-j}$ and is therefore impossible. Therefore, we have $\left(a_{j}, y_{-j}\right) \in \mathscr{F}$ and $\left(a_{j}, x_{-j}\right) \in \mathscr{A}$.

Conversely, suppose that $\left(a_{j}, x_{-j}\right) \in \mathscr{A}$ and $\left(a_{j}, y_{-j}\right) \in \mathscr{F}$, for some $a_{j} \in X_{j}$. This implies $x_{-j} \succsim^{(j)} y_{-j}$. Suppose now that $y_{-j} \succsim^{(j)} x_{-j}$, so that $x_{-j} \sim^{(j)} y_{-j}$. Using (A-2), we have $\left(b_{j}, x_{-j}\right) \in \mathscr{F}$ and $\left(b_{j}, y_{-j}\right) \in \mathscr{F}$, for some $b_{j} \in X_{j}$. Using $(n-1)$-thin, this implies $x_{-j} \sim_{-j} y_{-j}$. This contradicts the fact that $\left(a_{j}, x_{-j}\right) \in \mathscr{A}$ and $\left(a_{j}, y_{-j}\right) \in \mathscr{F}$.

Part 4. Suppose that, for some $x_{i}, y_{i} \in X_{i}$ and some $a_{-i j}, b_{-i j} \in X_{-i j}$, $\left(x_{i}, a_{-i j}\right) \succsim^{(j)}\left(x_{i}, b_{-i j}\right)$ and $\left(y_{i}, b_{-i j}\right) \succ^{(j)}\left(y_{i}, a_{-i j}\right)$. Using the definition of $\succsim^{(j)}$, $\left(x_{i}, a_{-i j}\right) \succsim^{(j)}\left(x_{i}, b_{-i j}\right)$ implies $\left(c_{j}, x_{i}, a_{-i j}\right) \in \mathscr{A} \mathscr{F}$ and $\left(c_{j}, x_{i}, b_{-i j}\right) \in \mathscr{F} \mathscr{U}$, for some $c_{j} \in X_{j}$. Using (A-3), $\left(y_{i}, b_{-i j}\right) \succ^{(j)}\left(y_{i}, a_{-i j}\right)$ implies $\left(d_{j}, y_{i}, b_{-i j}\right) \in \mathscr{A}$ and $\left(d_{j}, y_{i}, a_{-i j}\right) \in \mathscr{F}$, for some $d_{j} \in X_{j}$. Using $(n-2)$-linear and $(n-2)$-thin, this implies $b_{-i j} \succ_{-i j} a_{-i j}$. But $\left(c_{j}, x_{i}, a_{-i j}\right) \in \mathscr{A} \mathscr{F}$ and $b_{-i j} \succ_{-i j} a_{-i j}$ imply, using $(n-2)$-linear and $(n-2)$-thin, $\left(c_{j}, x_{i}, b_{-i j}\right) \in \mathscr{A}$, a contradiction.

Part 5. Let $y_{-j} \in X_{-j}$ and $a_{-i j} \in X_{-i j}$. We must show that $y_{-j} \sim^{(j)}\left(b_{i}, a_{-i j}\right)$, for some $b_{i} \in X_{i}$. Using unrestricted solvability on attribute $j$, we have $\left(a_{j}, y_{-j}\right) \in$ $\mathscr{F}$, for some $a_{j} \in X_{j}$. Using unrestricted solvability on any attribute $i \neq j$, we know that $\left(a_{j}, b_{i}, a_{-i j}\right) \in \mathscr{F}$, for some $b_{i} \in X_{i}$. The conclusion follows from (A-2).

Part 6. Because $\langle\mathscr{A}, \mathscr{F}, \mathscr{U}\rangle$ is non-degenerate, we know that $\mathscr{A} \neq \varnothing$. Let $x=\left(x_{j}, x_{i}, x_{-i j}\right) \in \mathscr{A}$. Take any attribute $i \neq j$. Using unrestricted solvability on $i \in N$, we have $\left(x_{j}, y_{i}, x_{-i j}\right) \in \mathscr{F}$, for some $y_{i} \in X_{i}$. Using (A-3), this implies $\left(x_{i}, x_{-i j}\right) \succ^{(j)}\left(y_{i}, x_{-i j}\right)$.

Part 7. Let $i \in N \backslash\{j\}$. Consider a standard sequence $\left\{x_{i}^{\tau} \in X_{i}: \tau \in\right.$ $\mathbb{T}\}$ for $\succsim^{(j)}$. Hence, there are $a_{-i j}, b_{-i j} \in X_{-i j}$ such that, for some $c_{i} \in X_{i}$, $\operatorname{Not}\left[\left(c_{i}, a_{-i j}\right) \sim^{(j)}\left(c_{i}, b_{-i j}\right)\right]$ and $\left(x_{i}^{\tau}, a_{-i j}\right) \sim^{(j)}\left(x_{i}^{\tau+1}, b_{-i j}\right)$, for all $\tau \in \mathbb{T}$. Using $(n-2)$-linear and $(n-2)$-thin, we know that $\operatorname{Not}\left[a_{-i j} \sim_{-i j} b_{-i j}\right]$. Using solvability, we can find $x_{j}^{\tau}, x_{j}^{\tau-1} \in X_{j}$ such that $\left(x_{i}^{\tau}, x_{j}^{\tau-1}, b_{-i j}\right) \in \mathscr{F},\left(x_{i}^{\tau}, x_{j}^{\tau}, a_{-i j}\right) \in \mathscr{F}$ and $\left(x_{i}^{\tau+1}, x_{j}^{\tau}, b_{-i j}\right) \in \mathscr{F}$. Hence, $\left\{x_{i}^{\tau} \in X_{i}, x_{j}^{\tau} \in X_{j},: \tau \in \mathbb{T}\right\}$, is a diagonal standard sequence for $\langle\mathscr{A}, \mathscr{F}, \mathscr{U}\rangle$.

Suppose that there are $\underline{x}_{i}, \bar{x}_{i} \in X_{i}$ such that, for all $\tau \in \mathbb{T}, \bar{x}_{i} \succ_{i}^{(j)} x_{i}^{\tau}$ and $x_{i}^{\tau} \succ_{i}^{(j)} \underline{x}_{i}$, where $\succsim_{i}^{(j)}$ is the marginal relation induced by $\succsim^{(j)}$ on $X_{i}$. Using $(\mathrm{A}-1)$, this clearly implies $\bar{x}_{i} \succ_{i} x_{i}^{\tau}$ and $x_{i}^{\tau} \succ_{i} \underline{x}_{i}$, so that the diagonal standard sequence 
for $\langle\mathscr{A}, \mathscr{F}, \mathscr{U}\rangle$ is strictly bounded on $i \in N$. Using the Archimedean condition for $\langle\mathscr{A}, \mathscr{F}, \mathscr{U}\rangle$, we know that this sequence must be finite.

Part 8. Suppose that $n=3$ and let $N=\{i, j, k\}$. Suppose that $\left(x_{i}, x_{k}\right) \sim^{(j)}$ $\left(y_{i}, y_{k}\right)$ and $\left(y_{i}, z_{k}\right) \sim^{(j)}\left(z_{i}, x_{k}\right)$. Using Part 4 , we know that $\left(x_{i}, x_{k}, a_{j}\right) \in \mathscr{F}$ and $\left(y_{i}, z_{k}, b_{j}\right) \in \mathscr{F}$, for some $a_{j}, b_{j} \in X_{j}$. Using Part 1 , we have $\left(x_{i}, x_{k}\right) \sim_{i k}\left(y_{i}, y_{k}\right)$ and $\left(y_{i}, z_{k}\right) \sim_{i k}\left(z_{i}, x_{k}\right)$. Using Thomsen, we therefore obtain $\left(x_{i}, z_{k}\right) \sim_{i k}\left(z_{i}, y_{k}\right)$. The conclusion follows from Part 1.

Part 9. Suppose that $\left\langle u_{i}\right\rangle_{i \neq j}$ is an additive representation of $\succsim^{(j)}$. Let $x_{j} \in X_{j}$. Using unrestricted solvability on any attribute $i$ other than $j$, we can always find a $a_{-j} \in X_{-j}$ such that $\left(x_{j}, a_{-j}\right) \in \mathscr{F}$. Now, define $u_{j}$ letting, for all $x_{j} \in X_{j}$,

$$
u_{j}\left(x_{j}\right)=-\sum_{i \neq j} u_{i}\left(a_{i}\right) \text { if }\left(x_{j}, a_{-j}\right) \in \mathscr{F} .
$$

It is easy to see that $u_{j}$ is well-defined. Indeed if $\left(x_{j}, a_{-j}\right) \in \mathscr{F}$ and $\left(x_{j}, b_{-j}\right) \in \mathscr{F}$, (A-2) implies $a_{-j} \sim^{(j)} b_{-j}$, so that:

$$
\sum_{i \neq j} u_{i}\left(a_{i}\right)=\sum_{i \neq j} u_{i}\left(b_{i}\right)
$$

Let us now show that such a function $u_{j}$ together with the functions $\left\langle u_{i}\right\rangle_{i \neq j}$ give an additive representation for $\langle\mathscr{A}, \mathscr{F}, \mathscr{U}\rangle$.

If $\left(x_{j}, x_{-j}\right) \in \mathscr{F}$, then, by construction, we have $u_{j}\left(x_{j}\right)+\sum_{i \neq j} u_{i}\left(x_{i}\right)=0$.

Suppose that $\left(x_{j}, x_{-j}\right) \in \mathscr{A}$. Using unrestricted solvability on any attribute other than $j$, we know that $\left(x_{j}, a_{-j}\right) \in \mathscr{F}$, for some $a_{-j} \in X_{-j}$. Hence, we have:

$$
u_{j}\left(x_{j}\right)=-\sum_{i \neq j} u_{i}\left(a_{i}\right)
$$

Using (A-3), $\left(x_{j}, x_{-j}\right) \in \mathscr{A}$ and $\left(x_{j}, a_{-j}\right) \in \mathscr{F}$ imply $x_{-j} \succ^{(j)} a_{-j}$, so that:

$$
\sum_{i \neq j} u_{i}\left(x_{i}\right)>\sum_{i \neq j} u_{i}\left(a_{i}\right)
$$

which implies

$$
u_{j}\left(x_{j}\right)+\sum_{i \neq j} u_{i}\left(x_{i}\right)>0 .
$$

That $\left(x_{j}, x_{-j}\right) \in \mathscr{U}$ implies $u_{j}\left(x_{j}\right)+\sum_{i \neq j} u_{i}\left(x_{i}\right)<0$ is shown similarly. Hence we have built an additive representation of $\langle\mathscr{A}, \mathscr{F}, \mathscr{U}\rangle$.

\section{A.2 Proof of Proposition 1: existence}

Using Parts $1-8$ of Lemma A-1, we know that $\succsim^{(j)}$ is an independent weak order satisfying unrestricted solvability and the Archimedean condition. Furthermore, 
we know that there is at least one essential attribute for $\succsim^{(j)}$ and that, if $n=3$, $\succsim^{(j)}$ satisfies the Thomsen condition. We can therefore use the classical theorems of conjoint measurement (see Krantz et al., 1971, Ch. 6) ${ }^{2}$ to obtain an additive representation for $\succsim^{(j)}$. The conclusion follows from Part 9 of Lemma A-1.

\section{A.3 Proof of Proposition 1: uniqueness}

It is clear that if $\left\langle u_{i}\right\rangle_{i \in N}$ is an additive representation using the threshold 0 for $\mathscr{F}$ of $\langle\mathscr{A}, \mathscr{F}, \mathscr{U}\rangle$, then $\left\langle\alpha u_{i}+\beta_{i}\right\rangle_{i \in N}$ with $\alpha>0$ and $\sum_{i=1}^{n} \beta_{i}=0$ will also be an additive representation with the same threshold.

Let $\left\langle u_{i}\right\rangle_{i \in N}$ be any additive representation of $\langle\mathscr{A}, \mathscr{F}, \mathscr{U}\rangle$. Let us show that $\left\langle u_{i}\right\rangle_{i \neq j}$ must be an additive representation of $\succsim^{(j)}$. Suppose that $x_{-j} \sim^{(j)} y_{-j}$. Using Part 2 of Lemma A-1, we must have $\sum_{i \neq j} u_{i}\left(x_{i}\right)=\sum_{i \neq j} u_{i}\left(y_{i}\right)$. Similarly, using Part 3 of Lemma A-1, $x_{-j} \succ^{(j)} y_{-j}$ implies $\sum_{i \neq j} u_{i}\left(x_{i}\right)>\sum_{i \neq j} u_{i}\left(y_{i}\right)$. Hence, any additive representation of $\langle\mathscr{A}, \mathscr{F}, \mathscr{U}\rangle$ must also be an additive representation of $\succsim^{(j)}$. Conversely, the proof of Part 9 of Lemma A-1 has shown that, given any additive representation for $\succsim^{(j)}$, we can obtain an additive representation for $\langle\mathscr{A}, \mathscr{F}, \mathscr{U}\rangle$ that uses the same functions for $i \neq j$.

Because $\succsim^{(j)}$ satisfies all conditions the classical theorems of conjoint measurement (see Krantz et al., 1971, Ch. 6), we know that any two additive representations $\left\langle u_{i}\right\rangle_{i \neq j}$ and $\left\langle v_{i}\right\rangle_{i \neq j}$ must be such that

$$
v_{i}\left(x_{i}\right)=\alpha u_{i}\left(x_{i}\right)+\beta_{i} .
$$

with $\alpha>0$.

Using unrestricted solvability on any attribute distinct from $j$, for all $x_{j} \in X_{j}$, we have $\left(x_{j}, y_{-j}\right) \in \mathscr{F}$, for some $y_{-j} \in X_{-j}$. This implies that if $\left\langle u_{i}\right\rangle_{i \in N}$ and $\left\langle v_{i}\right\rangle_{i \in N}$ are two representations of $\langle\mathscr{A}, \mathscr{F}, \mathscr{U}\rangle$, for all $x_{j} \in X_{j}$, we have

$$
\begin{gathered}
u_{j}\left(x_{j}\right)=-\sum_{i \neq j} u_{i}\left(y_{i}\right), \\
v_{j}\left(x_{j}\right)=-\sum_{i \neq j} v_{i}\left(y_{i}\right)=-\sum_{i \neq j}\left[\alpha u_{i}\left(y_{i}\right)+\beta_{i}\right],
\end{gathered}
$$

where $y_{-j} \in X_{-j}$ is such that $\left(x_{j}, y_{-j}\right) \in \mathscr{F}$. Therefore, we obtain $v_{j}=\alpha u_{j}-$ $\sum_{i \neq j} \beta_{i}$. Hence, the two sets of functions will be such that, for all $i \in N, v_{i}=$ $\alpha u_{i}+\beta_{i}$ with $\alpha>0$ and $\sum_{i=1}^{n} \beta_{i}=0$.

\footnotetext{
${ }^{2}$ More precisely, we make use of variants of Krantz et al. (1971, Theorem 6.2, page 257) (when $n=3$ ) and of Krantz et al. (1971, Theorem 6.13, page 302) (when $n \geq 4$ ) in which restricted solvability is replaced by unrestricted solvability. In this case, if there is at least one essential attribute, then all attributes are essential.
} 


\section{B Refining conditions}

It is easy to check that the only consequences of strong linearity used in the proof of Lemma A-1 are 1-linear, $(n-2)$-linear and $(n-1)$-linear. Similarly, the only consequences of strong thinness that are used are 1-thin, $(n-2)$-thin and $(n-1)$-thin. Therefore these conditions can fully replace strong linearity and strong thinness in the statement of Proposition 1. Working with groups of $n-1$ or $n-2$ attributes is not particularly intuitive however. We show below how it is possible to work only with singletons and pairs.

\section{B.1 Proof of Theorem 1}

We start with some preliminary results. The proof of the following lemma follows directly from the definition of linearity.

\section{Lemma A-2}

1. $\langle\mathscr{A}, \mathscr{F}, \mathscr{U}\rangle$ satisfies $\mathscr{A}$-linear $_{I}$ iff it satisfies $\mathscr{A}$-linear $-I$,

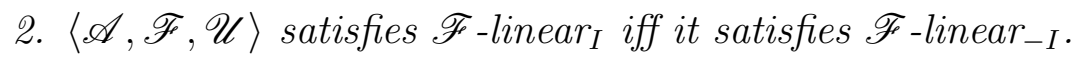

We have:

\section{Lemma A-3}

Let $\langle\mathscr{A}, \mathscr{F}, \mathscr{U}\rangle$ be an ordered covering satisfying $\mathscr{A}$-linear, $\mathscr{F}_{I}$-linear $_{I}$, thin th $_{I}$ and unrestricted solvability. Then $\langle\mathscr{A}, \mathscr{F}, \mathscr{U}\rangle$ satisfies $\mathscr{A} \mathscr{F}$-linear ${ }_{I}$.

\section{PROOF}

Suppose that $\mathscr{A} \mathscr{F}$-linear ${ }_{I}$ is violated, so that $\left(x_{I}, a_{-I}\right) \in \mathscr{A},\left(y_{I}, b_{-I}\right) \in \mathscr{F}$, $\left(y_{I}, a_{-I}\right) \notin \mathscr{A}$, and $\left(x_{I}, b_{-I}\right) \notin \mathscr{A} \mathscr{F}$.

Using unrestricted solvability, we can find a $c_{-I} \in X_{-I}$ such that $\left(x_{I}, c_{-I}\right) \in$ $\mathscr{F}$. Using $\mathscr{F}$-linear $,,\left(x_{I}, c_{-I}\right) \in \mathscr{F},\left(y_{I}, b_{-I}\right) \in \mathscr{F}$, and $\left(x_{I}, b_{-I}\right) \notin \mathscr{A} \mathscr{F}$ imply $\left(y_{I}, c_{-I}\right) \in \mathscr{A} \mathscr{F}$. Suppose first that $\left(y_{I}, c_{-I}\right) \in \mathscr{F}$. Since $\left(x_{I}, c_{-I}\right) \in \mathscr{F}, \operatorname{thin}_{I}$ implies that $x_{I} \sim_{I} y_{I}$, contradicting the fact that $\left(x_{I}, a_{-I}\right) \in \mathscr{A}$ and $\left(y_{I}, a_{-I}\right) \notin \mathscr{A}$. Suppose now that $\left(y_{I}, c_{-I}\right) \in \mathscr{A}$. Since $\left(x_{I}, a_{-I}\right) \in \mathscr{A}, \mathscr{A}$-linear $\operatorname{limplies}_{I}$ either $\left(x_{I}, c_{-I}\right) \in \mathscr{A}$ or $\left(y_{I}, a_{-I}\right) \in \mathscr{A}$, a contradiction.

\section{Lemma A-4}

An ordered covering $\langle\mathscr{A}, \mathscr{F}, \mathscr{U}\rangle$ satisfies $\mathscr{A} \mathscr{F}$-linear and thin $_{I}$ iff it satisfies $\mathscr{A} \mathscr{F}$-linear-I and thin $-I$.

\section{ProOF}

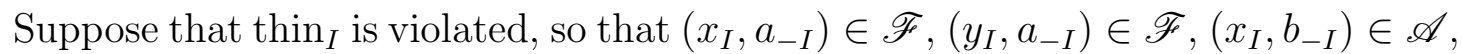
$\left(y_{I}, b_{-I}\right) \in \mathscr{F} \mathscr{U}$. If $\left(y_{I}, b_{-I}\right) \in \mathscr{F}$, thin ${ }_{-I}$ and $\left(y_{I}, a_{-I}\right) \in \mathscr{F}$ imply that $a_{-I} \sim_{-I}$ $b_{-I}$, contradicting the fact that $\left(x_{I}, a_{-I}\right) \in \mathscr{F}$ and $\left(x_{I}, b_{-I}\right) \in \mathscr{A}$. Suppose now 
that $\left(y_{I}, b_{-I}\right) \in \mathscr{U}$. Using $\mathscr{A} \mathscr{F}$-linear $-I,\left(x_{I}, b_{-I}\right) \in \mathscr{A}$ and $\left(y_{I}, a_{-I}\right) \in \mathscr{F}$ imply either $\left(x_{I}, a_{-I}\right) \in \mathscr{A}$ or $\left(y_{I}, b_{-I}\right) \in \mathscr{A} \mathscr{F}$, a contradiction.

Suppose now that $\mathscr{A} \mathscr{F}$-linear $I$ is violated, so that we have $\left(x_{I}, a_{-I}\right) \in \mathscr{A}$, $\left(y_{I}, b_{-I}\right) \in \mathscr{F},\left(y_{I}, a_{-I}\right) \in \mathscr{F} \mathscr{U}$ and $\left(x_{I}, b_{-I}\right) \in \mathscr{U}$. If $\left(y_{I}, a_{-I}\right) \in \mathscr{F}$, thin ${ }_{-I}$ and $\left(y_{I}, b_{-I}\right) \in \mathscr{F}$ imply that that $a_{-I} \sim_{-I} b_{-I}$, contradicting the fact that $\left(x_{I}, a_{-I}\right) \in$ $\mathscr{A}$ and $\left(x_{I}, b_{-I}\right) \in \mathscr{U}$. Suppose now that $\left(y_{I}, a_{-I}\right) \in \mathscr{U}$. Using $\mathscr{A} \mathscr{F}$-linear $-I$, $\left(x_{I}, a_{-I}\right) \in \mathscr{A}$ and $\left(y_{I}, b_{-I}\right) \in \mathscr{F}$ imply either $\left(x_{I}, b_{-I}\right) \in \mathscr{A}$ or $\left(y_{I}, a_{-I}\right) \in \mathscr{A} \mathscr{F}$, a contradiction.

Proof of TheOREM 1

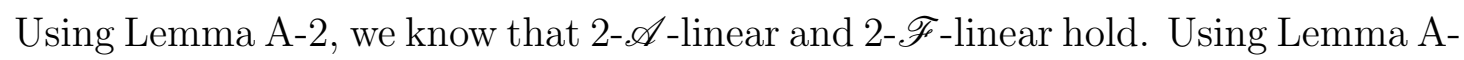

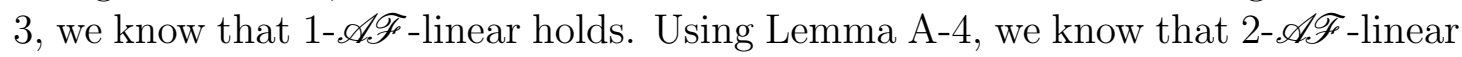
and 2-thin hold. The conclusion follows from Proposition 1.

\section{B.2 Proof of Theorem 2}

We start with some preliminary results.

\section{Lemma A-5}

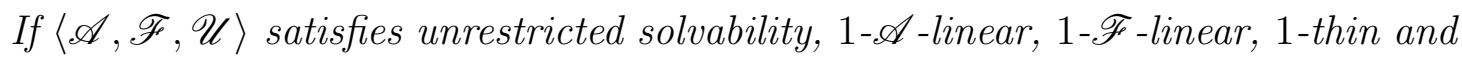

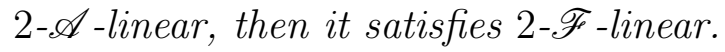

\section{PROOF}

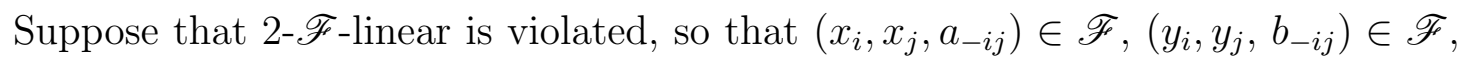
$\left(y_{i}, y_{j}, a_{-i j}\right) \in \mathscr{U},\left(x_{i}, x_{j}, b_{-i j}\right) \in \mathscr{U}$. Using Lemma A-3, we know that 1- $\mathscr{A} \mathscr{F}$-linear holds, so that $\succsim_{i}$ is complete, for all $i \in N$. It is clearly impossible that we have $x_{i} \succsim_{i} y_{i}$ and $x_{j} \succsim_{j} y_{j}$. Indeed, $\left(y_{i}, y_{j}, b_{-i j}\right) \in \mathscr{F}, x_{i} \succsim_{i} y_{i}$ and $x_{j} \succsim_{j} y_{j}$ would imply $\left(x_{i}, x_{j}, b_{-i j}\right) \in \mathscr{A} \mathscr{F}$, a contradiction. Similarly, it is impossible that $y_{i} \succsim_{i} x_{i}$ and $y_{j} \succsim_{j} x_{j}$.

Suppose henceforth that $x_{i} \succ_{i} y_{i}$ and $y_{j} \succsim_{j} x_{j}$, the other case being dealt with similarly. Because $\left(y_{i}, y_{j}, b_{-i j}\right) \in \mathscr{F}$ and $x_{i} \succ_{i} y_{i}$, we obtain $\left(x_{i}, y_{j}, b_{-i j}\right) \in \mathscr{A}$. Using unrestricted solvability, we know that $\left(x_{i}, w_{j}, b_{-i j}\right) \in \mathscr{F}$, for some $w_{j} \in X_{j}$. Because $\left(x_{i}, x_{j}, b_{-i j}\right) \in \mathscr{U}$, we must have $w_{j} \succ_{j} x_{j}$. Hence, from $\left(x_{i}, x_{j}, a_{-i j}\right) \in \mathscr{F}$, we obtain $\left(x_{i}, w_{j}, a_{-i j}\right) \in \mathscr{A}$. Similarly, using unrestricted solvability, we can find a $z_{j} \in X_{j}$ such that $\left(y_{i}, z_{j}, a_{-i j}\right) \in \mathscr{F}$. Because $\left(y_{i}, y_{j}, a_{-i j}\right) \in \mathscr{U}$, it must be true that $z_{j} \succ_{j} y_{j}$. Using $\left(y_{i}, y_{j}, b_{-i j}\right) \in \mathscr{F}$, we obtain $\left(y_{i}, z_{j}, b_{-i j}\right) \in \mathscr{A}$. Using 2- $\mathscr{A}$-linear, $\left(x_{i}, w_{j}, a_{-i j}\right) \in \mathscr{A}$ and $\left(y_{i}, z_{j}, b_{-i j}\right) \in \mathscr{A}$ imply either $\left(y_{i}, z_{j}, a_{-i j}\right) \in \mathscr{A}$ or $\left(x_{i}, w_{j}, b_{-i j}\right) \in \mathscr{A}$, a contradiction.

\section{Lemma A-6}

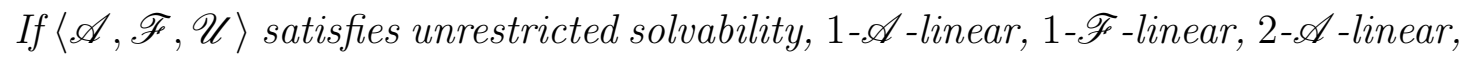
1 -thin and 2 -thin, then it satisfies $(n-2)$-linear, $(n-1)$-linear, $(n-2)$-thin and $(n-1)$-thin. 


\section{PROOF}

Using Lemma A-5, we know that 2- $\mathscr{F}$-linear holds. Using Lemma A-2, we know that $(n-2)-\mathscr{A}$-linear, $(n-1)-\mathscr{A}$-linear, $(n-2)-\mathscr{F}$-linear and $(n-1)$ - $\mathscr{F}$-linear

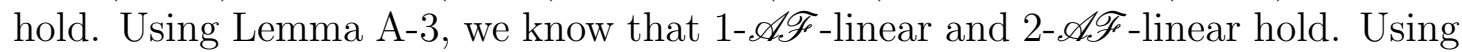
Lemma A-4, we know that $(n-2)$ - $\mathscr{A} \mathscr{F}$-linear, $(n-1)$ - $\mathscr{A} \mathscr{F}$-linear, $(n-2)$-thin and $(n-1)$-thin hold.

\section{Lemma A-7}

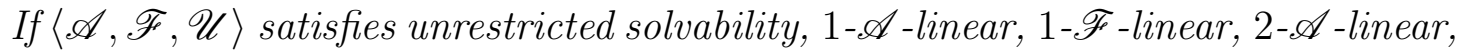
1-thin and the Archimedean condition, then it is 2-thin.

\section{PROOF}

Suppose that 2 -thin is violated, so that $\left(x_{i}, x_{j}, a_{-i j}\right) \in \mathscr{F},\left(y_{i}, y_{j}, a_{-i j}\right) \in \mathscr{F}$, $\left(x_{i}, x_{j}, b_{-i j}\right) \in \mathscr{A}$, and $\left(y_{i}, y_{j}, b_{-i j}\right) \in \mathscr{F} \mathscr{U}$. Using Lemma A-3, we know that 1$\mathscr{A} \mathscr{F}$-linear holds. Using Lemma A-5, we know that 2- $\mathscr{F}$-linear holds. Note that it is clearly impossible that $a_{-i j} \sim_{-i j} b_{-i j}$.

Suppose first that $\left(y_{i}, y_{j}, b_{-i j}\right) \in \mathscr{U}$. Using unrestricted solvability, we have $\left(z_{i}, x_{j}, b_{-i j}\right) \in \mathscr{F}$, for some $z_{i} \in X_{i}$. Because $\left(x_{i}, x_{j}, b_{-i j}\right) \in \mathscr{A}$, we must have that $x_{i} \succ_{i} z_{i}$. Now, $\left(x_{i}, x_{j}, a_{-i j}\right) \in \mathscr{F}$ and $x_{i} \succ_{i} z_{i}$ imply $\left(z_{i}, x_{j}, a_{-i j}\right) \in \mathscr{U}$. Using 2$\mathscr{F}$-linear, $\left(z_{i}, x_{j}, b_{-i j}\right) \in \mathscr{F}$ and $\left(y_{i}, y_{j}, a_{-i j}\right) \in \mathscr{F}$ imply either $\left(z_{i}, x_{j}, a_{-i j}\right) \in \mathscr{A} \mathscr{F}$ or $\left(y_{i}, y_{j}, b_{-i j}\right) \in \mathscr{A} \mathscr{F}$, a contradiction.

Suppose now that $\left(y_{i}, y_{j}, b_{-i j}\right) \in \mathscr{F}$. It is easy to see that we cannot have $x_{i} \sim_{i} y_{i}$ and $x_{j} \sim_{j} y_{j}$. Indeed, using 1-linear and 1-thin, $\left(x_{i}, x_{j}, b_{-i j}\right) \in \mathscr{A}$ would imply $\left(y_{i}, y_{j}, b_{-i j}\right) \in \mathscr{A}$. Similarly, it is impossible to have $\left[x_{i} \succsim_{i} y_{i}\right.$ and $\left.x_{j} \succ_{j} y_{j}\right]$ or $\left[y_{i} \succsim_{i} x_{i}\right.$ and $\left.y_{j} \succ_{j} x_{j}\right]$. Indeed, in the first case, $\left(y_{i}, y_{j}, a_{-i j}\right) \in \mathscr{F}$ and $x_{i} \succsim_{i} y_{i}$ would imply $\left(x_{i}, y_{j}, a_{-i j}\right) \in \mathscr{A} \mathscr{F}$. Using $x_{j} \succ_{j} y_{j}$, we would obtain $\left(x_{i}, x_{j}, a_{-i j}\right) \in$ $\mathscr{A}$.

Suppose henceforth that $x_{i} \succ_{i} y_{i}$ and $y_{j} \succ_{j} x_{j}$, the other case being symmetric. Using $\left(y_{i}, y_{j}, b_{-i j}\right) \in \mathscr{F}$ and $y_{j} \succ_{j} x_{j}$, we obtain $\left(y_{i}, x_{j}, b_{-i j}\right) \in \mathscr{U}$. Using unrestricted solvability, we can find a $z_{i} \in X_{i}$ such that $\left(z_{i}, x_{j}, b_{-i j}\right) \in \mathscr{F}$. Because we know that $\left(x_{i}, x_{j}, b_{-i j}\right) \in \mathscr{A},\left(z_{i}, x_{j}, b_{-i j}\right) \in \mathscr{F}$ and $\left(y_{i}, x_{j}, b_{-i j}\right) \in \mathscr{U}$, we must have $x_{i} \succ_{i} z_{i} \succ_{i} y_{i}$. Using $\left(x_{i}, x_{j}, a_{-i j}\right) \in \mathscr{F}$ and $x_{i} \succ_{i} z_{i}$, we obtain $\left(z_{i}, x_{j}, a_{-i j}\right) \in \mathscr{U}$. Similarly, $\left(y_{i}, y_{j}, a_{-i j}\right) \in \mathscr{F}$ and $z_{i} \succ_{i} y_{i}$ imply $\left(z_{i}, y_{j}, a_{-i j}\right) \in \mathscr{A}$. Using unrestricted solvability, we can find a $z_{j} \in X_{j}$ such that $\left(z_{i}, z_{j}, a_{-i j}\right) \in \mathscr{F}$. Because $\left(z_{i}, y_{j}, a_{-i j}\right) \in \mathscr{A}$ and $\left(z_{i}, x_{j}, a_{-i j}\right) \in \mathscr{U}$, we must have $y_{j} \succ_{j} z_{j} \succ_{j} x_{j}$. Since $\left(z_{i}, x_{j}, b_{-i j}\right) \in \mathscr{F}$ and $z_{j} \succ_{j} x_{j}$, we obtain $\left(z_{i}, z_{j}, b_{-i j}\right) \in \mathscr{A}$. 
We have started with the hypothesis that

$$
\begin{aligned}
& x_{i} \succ_{i} y_{i}, y_{j} \succ_{j} x_{j}, \\
& \left(y_{i}, y_{j}, a_{-i j}\right) \in \mathscr{F}, \\
& \left(y_{i}, y_{j}, b_{-i j}\right) \in \mathscr{F}, \\
& \left(x_{i}, x_{j}, a_{-i j}\right) \in \mathscr{F}, \\
& \left(x_{i}, x_{j}, b_{-i j}\right) \in \mathscr{A} .
\end{aligned}
$$

We have shown that we can find $z_{i} \in X_{i}$ and $z_{j} \in X_{j}$ such that

$$
\begin{gathered}
x_{i} \succ_{i} z_{i} \succ_{i} y_{i}, y_{j} \succ_{j} z_{j} \succ_{j} x_{j}, \\
\left(z_{i}, z_{j}, a_{-i j}\right) \in \mathscr{F}, \\
\left(z_{i}, z_{j}, b_{-i j}\right) \in \mathscr{A} .
\end{gathered}
$$

Hence, we can iterate the above reasoning with $z_{i}$ and $z_{j}$ playing the roles of $x_{i}$ and $x_{j}$. Furthermore, we know that $\left(z_{i}, x_{j}, b_{-i j}\right) \in \mathscr{F}$.

Rename $x_{i}$ and $x_{j}$ as $w_{i}^{1}$ and $w_{j}^{1}$ and $z_{i}$ and $z_{j}$ as $w_{i}^{2}$ and $w_{j}^{2}$. We have

$$
\begin{aligned}
& \left(w_{i}^{1}, w_{j}^{1}, a_{-i j}\right) \in \mathscr{F}, \\
& \left(w_{i}^{2}, w_{j}^{1}, b_{-i j}\right) \in \mathscr{F}, \\
& \left(w_{i}^{2}, w_{j}^{2}, a_{-i j}\right) \in \mathscr{F},
\end{aligned}
$$

which shows that $w_{i}^{1}, w_{i}^{2}, w_{j}^{2}, w_{j}^{2}$ is a diagonal standard sequence on $i, j$, since we know that we do not have $a_{-i j} \sim_{-i j} b_{-i j}$.

The iteration of the above process will lead to build an infinite diagonal standard sequence. It is clear that this diagonal standard sequence is strictly bounded on $j$ by $y_{j}$ and $x_{j}$. This violates the Archimedean condition.

\section{Proof of TheOREM 2}

Using Lemma A-7, we know that 2-thin holds. The proof therefore follows from Lemma A-6, Proposition 1 and the observations at the beginning of this section.

We discuss below the independence of the conditions used in Theorems 1 and 2 .

\section{B.3 Examples}

When $n=3$, Theorem 1 uses, on top of non-degeneracy and unrestricted solvability, five necessary condition for an additive representation: 1- $\mathscr{A}$-linear, 1- $\mathscr{F}$-linear and 1-thin, the Thomsen condition and the Archimedean condition. Let us show that none of these five conditions can be dropped. 


\section{Example A-1 (1-thin)}

Take $X=\mathbb{R}^{3}$ and let

$$
\begin{aligned}
x \in \mathscr{A} \mathscr{F} & \Leftrightarrow x_{1}+x_{2}+x_{3} \geq-1, \\
x \in \mathscr{F} \mathscr{U} & \Leftrightarrow x_{1}+x_{2}+x_{3} \leq 1 .
\end{aligned}
$$

It is easy to see that this covering satisfies $1-\mathscr{A}$-linear, 1- $\mathscr{F}$-linear, unrestricted solvability, Thomsen and the Archimedean condition. It clearly violates 1 -thinness. $\diamond$

\section{Example A-2 (Thomsen condition)}

Let $X=\mathbb{R}^{3}$ and consider the ordered covering such that:

$$
\begin{aligned}
& x \in \mathscr{A} \Leftrightarrow x_{1}+x_{2}+\min \left(x_{1}, x_{2}\right)+x_{3}>0, \\
& x \in \mathscr{F} \Leftrightarrow x_{1}+x_{2}+\min \left(x_{1}, x_{2}\right)+x_{3}=0 .
\end{aligned}
$$

It is easy to check that this covering is $1-\mathscr{A}$-linear, $1-\mathscr{F}$-linear and 1 -thin. It satisfies unrestricted solvability as well as the Archimedean condition. The Thomsen condition is violated however since we have $(18,0,-18) \in \mathscr{F},(6,6,-18) \in \mathscr{F}$, $(30,0,-30) \in \mathscr{F},(6,18,-30) \in \mathscr{F}$ and $(30,6,-36) \in \mathscr{F}$ but $(18,18,-36) \in \mathscr{A} . \diamond$

\section{Example A-3 (1- $\mathscr{A}$-linear)}

Take $X=(0,1)^{3}$ and let

$$
\begin{aligned}
& x \in \mathscr{A} \Leftrightarrow\left\{\begin{array}{l}
x_{1}+x_{2}+x_{3}>1 / 2, \\
\text { and } \\
x_{1}+x_{2}+x_{3} \neq 1,
\end{array}\right. \\
& x \in \mathscr{F} \Leftrightarrow x_{1}+x_{2}+x_{3}=1 .
\end{aligned}
$$

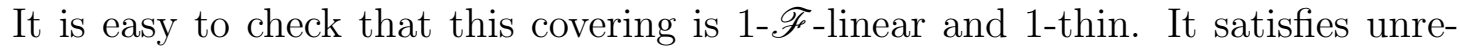
stricted solvability as well as the Archimedean condition and the Thomsen condition. Condition 1- $\mathscr{A}$-linear is violated since, for instance, $(1 / 2,1 / 4,0) \in \mathscr{A}$, $(1 / 4,1 / 2,0) \in \mathscr{A},(1 / 4,1 / 4,0) \in \mathscr{F} \mathscr{U}$ and $(1 / 2,1 / 2,0) \in \mathscr{F} \mathscr{U}$.

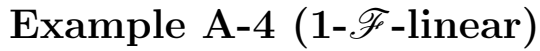

Take $X=(0,1)^{3}$ and let

$$
\begin{aligned}
& x \in \mathscr{A} \Leftrightarrow x_{1}+x_{2}+x_{3}>3 / 2, \\
& x \in \mathscr{F} \Leftrightarrow x_{1}+x_{2}+x_{3}=1 .
\end{aligned}
$$

It is easy to check that this covering $1-\mathscr{A}$-linear and 1 -thin. It satisfies unrestricted solvability as well as the Archimedean condition and the Thomsen condition. Condition 1- $\mathscr{F}$-linear is violated since, for instance, $(1 / 3,1 / 3,1 / 3) \in \mathscr{F}$, $(1 / 2,1 / 4,1 / 4) \in \mathscr{F},(1 / 3,1 / 4,1 / 4) \in \mathscr{U}$ and $(1 / 2,1 / 3,1 / 3) \in \mathscr{U}$. 
Our next example uses a binary relation satisfying all conditions of classical results of conjoint measurement, except the Archimedean condition. Krantz et al. (1971, Example 4, p. 261) give such an example. Unfortunately, we came to realize that this example is not fully satisfactory since it violates independence. Krantz (2009) suggested another example that has the desired properties. Let $\succsim$ be a binary relation on $X_{1} \times X_{2}$ and $\succsim^{\prime}$ be a binary relation on $A_{1} \times A_{2}$. We suppose that these two relations satisfy the classical conditions of additive conjoint measurement: weak ordering, independence, Thomsen and the Archimedean condition (p. 256 Krantz et al., 1971). We also suppose that these two relations satisfy unrestricted solvability. We now build a binary relation $\succsim^{\circ}$ on $\left[X_{1} \times A_{1}\right] \times\left[X_{2} \times A_{2}\right]$ as follows. We have:

$$
\begin{aligned}
& {\left[\left(x_{1}, a_{1}\right),\left(x_{2}, a_{2}\right)\right] \succ^{\circ}\left[\left(y_{1}, b_{1}\right),\left(y_{2}, b_{2}\right)\right] \Leftrightarrow} \\
& \quad\left\{\begin{array}{l}
\left(x_{1}, x_{2}\right) \succ\left(y_{1}, y_{2}\right) \text { or } \\
\left(x_{1}, x_{2}\right) \sim\left(y_{1}, y_{2}\right) \text { and }\left(a_{1}, a_{2}\right) \succ^{\prime}\left(b_{1}, b_{2}\right),
\end{array}\right.
\end{aligned}
$$

and

$$
\begin{gathered}
{\left[\left(x_{1}, a_{1}\right),\left(x_{2}, a_{2}\right)\right] \sim^{\circ}\left[\left(y_{1}, b_{1}\right),\left(y_{2}, b_{2}\right)\right] \Leftrightarrow} \\
\left(x_{1}, x_{2}\right) \sim\left(y_{1}, y_{2}\right) \text { and }\left(a_{1}, a_{2}\right) \sim^{\prime}\left(b_{1}, b_{2}\right) .
\end{gathered}
$$

A simple check shows that the relation ${ }^{\circ}$ on $\left[X_{1} \times A_{1}\right] \times\left[X_{2} \times A_{2}\right]$ satisfies weak ordering, independence, Thomsen and unrestricted solvability but violates the Archimedean condition. It is simple to extend this construction to cover the case of more than two attributes.

\section{Example A-5 (Archimedean condition)}

The above technique gives a general method to obtain a binary relation satisfying all conditions of classical results of conjoint measurement, except the Archimedean condition. Consider any such binary relation $\succsim^{\circ}$ on the set $X$. We denote by $\succsim_{I}^{\circ}$ the marginal relation that it induces on $X_{I}$.

Let $\left\{\beta_{i}^{\tau} \in X_{i}: \tau \in \mathbb{T}\right\}$ be an infinite standard sequence for $\succsim^{\circ}$ that is strictly bounded. Hence, there are $a_{-i}, b_{-i} \in X_{-i}$ such that $\operatorname{Not}\left[a_{-i} \sim_{-i}^{\circ} b_{-i}\right]$ and $\left(\beta_{i}^{\tau}, a_{-i}\right) \sim^{\circ}\left(\beta_{i}^{\tau+1}, b_{-i}\right)$, for all $\tau \in \mathbb{T}$. Furthermore, there are $\underline{\beta}_{i}, \bar{\beta}_{i} \in X_{i}$ such that, for all $\tau \in \mathbb{T}, \bar{\beta}_{i} \succ_{i}^{\circ} \beta_{i}^{\tau} \succ_{i}^{\circ} \underline{\beta}_{i}$, where $\succ_{i}^{\circ}$ denotes the asymmetric part of the marginal relation on $X_{i}$ induced by $\succsim^{\circ}$.

Using unrestricted solvability on any attribute other than $i$, we can find $c_{j}, d_{j} \in$ $X_{j}$ and $\alpha_{-i j} \in X_{-i j}$ such that $\left(c_{j}, \alpha_{-i j}\right) \sim_{-i}^{\circ} a_{-i}$ and $\left(d_{j}, \alpha_{-i j}\right) \sim_{-i}^{\circ} b_{-i}$. Hence, we have $\left(\beta_{i}^{\tau}, c_{j}, \alpha_{-i j}\right) \sim^{\circ}\left(\beta_{i}^{\tau+1}, d_{j}, \alpha_{-i j}\right)$, for all $\tau \in \mathbb{T}$.

Consider any attribute $k$ other than $i$ and $j$. Let $\alpha_{k}^{0}=\alpha_{k}$. Hence we have

$$
\left(\beta_{i}^{\tau}, c_{j}, \alpha_{k}^{0}, \alpha_{-i j k}\right) \sim^{\circ}\left(\beta_{i}^{\tau+1}, d_{j}, \alpha_{k}^{0}, \alpha_{-i j k}\right),
$$

for all $\tau \in \mathbb{T}$. 
Suppose w.l.o.g. that $1 \in \mathbb{T}$. Let us now build an ordered covering of $X$ letting, for all $x \in X$,

$$
\begin{aligned}
& x \in \mathscr{A} \Leftrightarrow x \succ^{\circ}\left(\beta_{i}^{1}, c_{j}, \alpha_{k}^{0}, \alpha_{-i j k}\right), \\
& x \in \mathscr{F} \Leftrightarrow x \sim^{\circ}\left(\beta_{i}^{1}, c_{j}, \alpha_{k}^{0}, \alpha_{-i j k}\right) .
\end{aligned}
$$

It is easy to check that this covering is strongly thin and strongly linear. It is clear that it satisfies unrestricted solvability as well as Thomsen.

By construction of the standard sequence for $\succsim^{\circ}$, we have $\left(\beta_{i}^{1}, c_{j}, \alpha_{k}^{0}, \alpha_{-i j k}\right) \sim^{\circ}$ $\left(\beta_{i}^{2}, d_{j}, \alpha_{k}^{0}, \alpha_{-i j k}\right)$. By construction of $\mathscr{F}$, we have $\left(\beta_{i}^{2}, d_{j}, \alpha_{k}^{0}, \alpha_{-i j k}\right) \in \mathscr{F}$. Because $\sim^{0}$ is reflexive, we also have $\left(\beta_{i}^{1}, c_{j}, \alpha_{k}^{0}, \alpha_{-i j}\right) \in \mathscr{F}$. We have therefore shown that

$$
\begin{gathered}
\left(\beta_{i}^{2}, d_{j}, \alpha_{k}^{0}, \alpha_{-i j k}\right) \in \mathscr{F}, \\
\left(\beta_{i}^{1}, c_{j}, \alpha_{k}^{0}, \alpha_{-i j}\right) \in \mathscr{F} .
\end{gathered}
$$

By definition of the standard sequence for $\succsim^{\circ}$, we know that $\left(\beta_{i}^{2}, c_{j}, \alpha_{k}^{0}, \alpha_{-i j k}\right) \sim^{\circ}$ $\left(\beta_{i}^{3}, d_{j}, \alpha_{k}^{0}, \alpha_{-i j k}\right)$. Using solvability on $k \in N$, we can find $\alpha_{k}^{-1} \in X_{k}$ such that $\left(\beta_{i}^{2}, c_{j}, \alpha_{k}^{-1}, \alpha_{-i j k}\right) \sim^{\circ}\left(\beta_{i}^{1}, c_{j}, \alpha_{k}^{0}, \alpha_{-i j k}\right)$. Hence, we know that $\left(\beta_{i}^{2}, c_{j}, \alpha_{k}^{-1}, \alpha_{-i j k}\right) \in$ $\mathscr{F}$. Let us show that we have $\left(\beta_{i}^{3}, d_{j}, \alpha_{k}^{-1}, \alpha_{-i j k}\right) \sim^{\circ}\left(\beta_{i}^{1}, c_{j}, \alpha_{k}^{0}, \alpha_{-i j k}\right)$, which will imply that $\left(\beta_{i}^{3}, d_{j}, \alpha_{k}^{-1}, \alpha_{-i j k}\right) \in \mathscr{F}$.

Suppose in contradiction with the thesis that $\left(\beta_{i}^{3}, d_{j}, \alpha_{k}^{-1}, \alpha_{-i j k}\right) \succ^{\circ}\left(\beta_{i}^{1}, c_{j}, \alpha_{k}^{0}\right.$, $\left.\alpha_{-i j k}\right)$, the opposite case being dealt with similarly. We have chosen $\alpha_{k}^{-1}$ in such a way that $\left(\beta_{i}^{2}, c_{j}, \alpha_{k}^{-1}, \alpha_{-i j k}\right) \sim^{\circ}\left(\beta_{i}^{1}, c_{j}, \alpha_{k}^{0}, \alpha_{-i j k}\right)$. Because $\left(\beta_{i}^{3}, d_{j}, \alpha_{k}^{-1}, \alpha_{-i j k}\right) \succ^{\circ}$ $\left(\beta_{i}^{1}, c_{j}, \alpha_{k}^{0}, \alpha_{-i j k}\right)$ and $\left(\beta_{i}^{2}, c_{j}, \alpha_{k}^{-1}, \alpha_{-i j k}\right) \sim^{\circ}\left(\beta_{i}^{1}, c_{j}, \alpha_{k}^{0}, \alpha_{-i j k}\right)$, we obtain $\left(\beta_{i}^{3}, d_{j}, \alpha_{k}^{-1}\right.$, $\left.\alpha_{-i j k}\right) \succ^{\circ}\left(\beta_{i}^{2}, c_{j}, \alpha_{k}^{-1}, \alpha_{-i j k}\right)$, since $\succsim^{\circ}$ is a weak order. Using the independence of $\succsim^{\circ}$, this implies $\left(\beta_{i}^{3}, d_{j}, \alpha_{k}^{0}, \alpha_{-i j k}\right) \succ^{\circ}\left(\beta_{i}^{2}, c_{j}, \alpha_{k}^{0}, \alpha_{-i j k}\right)$, a contradiction. Hence, we must have $\left(\beta_{i}^{3}, d_{j}, \alpha_{k}^{-1}, \alpha_{-i j k}\right) \sim^{\circ}\left(\beta_{i}^{1}, c_{j}, \alpha_{k}^{0}, \alpha_{-i j k}\right)$, which implies $\left(\beta_{i}^{3}, d_{j}, \alpha_{k}^{-1}, \alpha_{-i j k}\right) \in$ $\mathscr{F}$. We therefore know that

$$
\begin{aligned}
\left(\beta_{i}^{3}, d_{j}, \alpha_{k}^{-1}, \alpha_{-i j k}\right) & \in \mathscr{F}, \\
\left(\beta_{i}^{2}, c_{j}, \alpha_{k}^{-1}, \alpha_{-i j k}\right) & \in \mathscr{F} .
\end{aligned}
$$

Iterating the above reasoning, for all $\tau \in \mathbb{T}$, we will find $\alpha_{k}^{-\tau+1}$ such that

$$
\begin{gathered}
\left(\beta_{i}^{\tau+1}, d_{j}, \alpha_{k}^{-\tau+1}, \alpha_{-i j k}\right) \in \mathscr{F}, \\
\left(\beta_{i}^{\tau}, c_{j}, \alpha_{k}^{-\tau+1}, \alpha_{-i j k}\right) \in \mathscr{F} .
\end{gathered}
$$

This shows that to the infinite standard sequence for $\succsim^{\circ}\left\{\beta_{i}^{\tau}: \tau \in \mathbb{T}\right\}$ corresponds an infinite diagonal standard sequence on $i$ and $k$ for the ordered partition. It remains to show that this infinite diagonal standard sequence is strictly bounded.

We know that $\bar{\beta}_{i} \succ_{i}^{\circ} \beta_{i}^{\tau} \succ_{i}^{\circ} \underline{\beta}_{i}$. Furthermore, because $\left(\beta_{i}^{\tau}, c_{j}, \alpha_{k}^{-\tau+1}, \alpha_{-i j k}\right) \in$ $\mathscr{F}$, we have $\left(\beta_{i}^{\tau}, c_{j}, \alpha_{k}^{-\tau+1}, \alpha_{-i j k}\right) \sim^{\circ}\left(\beta_{i}^{1}, c_{j}, \alpha_{k}^{0}, \alpha_{-i j k}\right)$. This implies that $\left(\bar{\beta}_{i}, c_{j}, \alpha_{k}^{-\tau+1}\right.$, 
$\left.\alpha_{-i j k}\right) \succ^{\circ}\left(\beta_{i}^{1}, c_{j}, \alpha_{k}^{0}, \alpha_{-i j k}\right)$. Hence we know that $\left(\beta_{i}^{\tau}, c_{j}, \alpha_{k}^{-\tau+1}, \alpha_{-i j k}\right) \in \mathscr{F}$ and $\left(\bar{\beta}_{i}, c_{j}, \alpha_{k}^{-\tau+1}, \alpha_{-i j k}\right) \in \mathscr{A}$. This implies that $\bar{\beta}_{i} \succ_{i} \beta_{i}^{\tau}$. The proof that $\beta_{i}^{\tau} \succ_{i} \underline{\beta}_{i}$ is similar. Hence the infinite diagonal standard sequence on $i$ and $k$ is strictly bounded on $i$.

When $n \geq 4$, Theorem 2 uses, on top of non-degeneracy and unrestricted solvabili-

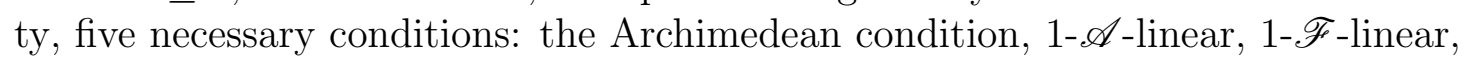
2 - $\mathscr{A}$-linear, 1 -thin. It is not difficult to adapt the above examples to show that none of the Archimedean condition, 1- $\mathscr{F}$-linear and 1-thin can be omitted from this proposition. The following example show that $2-\mathscr{A}$-linear cannot be omitted either.

Example A-6 (2- $\mathscr{A}$-linear)

Take $X=\mathbb{R}^{4}$ and let

$$
\begin{aligned}
& x \in \mathscr{A} \mathscr{F} \Leftrightarrow x_{1}+x_{2}+\min \left(x_{1}, x_{2}\right)+x_{3}+x_{4}+\min \left(x_{3}, x_{4}\right) \geq 0, \\
& x \in \mathscr{F} \mathscr{U} \Leftrightarrow x_{1}+x_{2}+\min \left(x_{1}, x_{2}\right)+x_{3}+x_{4}+\min \left(x_{3}, x_{4}\right) \leq 0 .
\end{aligned}
$$

It is easy to check that this covering satisfies unrestricted solvability, the Archi-

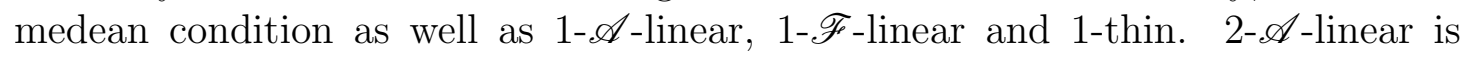
violated since, e.g., $(5,5,-4,-4) \in \mathscr{A},(-4,-4,5,5) \in \mathscr{A}$ but $(5,-4,5,-4) \in \mathscr{U}$ and $(-4,5,-4,5) \in \mathscr{U}$.

Unfortunately, we have been unable to show that condition 1- $\mathscr{A}$-linear cannot be omitted (for more details on this point, see Bouyssou and Marchant, 2009).

\section{Proofs with more than two categories}

\section{C.1 Proof of Theorem 3: existence}

Let $k \in\{1,2, \ldots, r-1\}$ be such that $\left\langle\Delta_{<}^{k}, C^{k} \cap C^{k+1}, \Delta_{>}^{k+1}\right\rangle$ satisfies the conditions mentioned in Part 2 of Proposition 3. Because $\left\langle C^{k}\right\rangle_{k \in R}$ is non-degenerate, generalized 1-thinness, $\Upsilon$-mixed-1-linearity, $\Delta$-Mixed-1-linearity and unrestricted solvability w.r.t. all frontiers hold, we know using Theorem 1 (if $n=3$ ) or Theorem 2 (if $n \geq 4$ ) that there is an additive representation of $\left\langle\Delta_{\leq}^{k}, C^{k} \cap C^{k+1}, \Delta_{\geq}^{k+1}\right\rangle$ such that

$$
\begin{array}{r}
x \in \Delta_{\geq}^{k+1} \Leftrightarrow \sum_{i=1}^{n} v_{i}^{(k)}\left(x_{i}\right)>0, \\
x \in C^{k} \cap C^{k+1} \Leftrightarrow \sum_{i=1}^{n} v_{i}^{(k)}\left(x_{i}\right)=0,
\end{array}
$$

for all $x \in X$.

Our strategy will be to show that this additive representation also gives an additive representation of $\left\langle C^{1}, C^{2}, \ldots, C^{r}\right\rangle$ in model $\left(A^{*}\right)$. 


\section{Step 1}

Let $h \in\{1,2, \ldots, r-1\}$ with $h \neq k$. Consider first any two alternatives $x, y \in$ $C^{h} \cap C^{h+1}$. Let us show that we must have $\sum_{i=1}^{n} v_{i}^{(k)}\left(x_{i}\right)=\sum_{i=1}^{n} v_{i}^{(k)}\left(y_{i}\right)$.

Using unrestricted solvability w.r.t. $C^{h} \cap C^{h+1}$, we can find $z_{1}^{2}, z_{1}^{3}, \ldots, z_{1}^{n} \in X_{1}$ such that

$$
\begin{gathered}
\left(z_{1}^{2}, y_{2}, x_{3}, x_{4}, x_{5}, \ldots, x_{n}\right) \in C^{h} \cap C^{h+1}, \\
\left(z_{1}^{3}, y_{2}, y_{3}, x_{4}, x_{5}, \ldots, x_{n}\right) \in C^{h} \cap C^{h+1}, \\
\left(z_{1}^{4}, y_{2}, y_{3}, y_{4}, x_{5}, \ldots, x_{n}\right) \in C^{h} \cap C^{h+1}, \\
\vdots \\
\left(z_{1}^{n}, y_{2}, y_{3}, y_{4}, y_{5}, \ldots, y_{n}\right) \in C^{h} \cap C^{h+1} .
\end{gathered}
$$

Using unrestricted solvability w.r.t. $C^{k} \cap C^{k+1}$, we know that we can find $w_{-12} \in$ $X_{-12}$ such that $\left(x_{1}, x_{2}, w_{-12}\right) \in C^{k} \cap C^{k+1}$. Because $\left(x_{1}, x_{2}, x_{-12}\right) \in C^{h} \cap C^{h+1}$, $\left(z_{1}^{2}, y_{2}, x_{-12}\right) \in C^{h} \cap C^{h+1}$ and $\left(x_{1}, x_{2}, w_{-12}\right) \in C^{k} \cap C^{k+1}$, parallelism implies that $\left(z_{1}^{2}, y_{2}, w_{-12}\right) \in C^{k} \cap C^{k+1}$. Using the additive representation for $\left\langle C_{\leq}^{k}, C_{\geq}^{k+1}\right\rangle$, we therefore know that:

$$
v_{1}^{(k)}\left(x_{1}\right)+v_{2}^{(k)}\left(x_{2}\right)=v_{1}^{(k)}\left(z_{1}^{2}\right)+v_{2}^{(k)}\left(y_{2}\right) .
$$

Let us now iterate the above reasoning. Using unrestricted solvability w.r.t. $C^{k} \cap$ $C^{k+1}$, we know that we can find $w_{-13} \in X_{-13}$ such that $\left(z_{1}^{2}, x_{3}, w_{-13}\right) \in C^{k} \cap$ $C^{k+1}$. Because $\left(z_{1}^{2}, y_{2}, x_{3}, x_{-123}\right) \in C^{h} \cap C^{h+1},\left(z_{1}^{3}, y_{2}, y_{3}, x_{-123}\right) \in C^{h} \cap C^{h+1}$ and $\left(z_{1}^{2}, x_{3}, w_{-13}\right) \in C^{k} \cap C^{k+1}$, parallelism implies that $\left(z_{1}^{3}, y_{3}, w_{-13}\right) \in C^{k} \cap C^{k+1}$. Using the additive representation for $\left\langle\Delta_{\leq}^{k-1}, C^{k} \cap C^{k+1}, \Delta_{\geq}^{k+1}\right\rangle$, we know that:

$$
v_{1}^{(k)}\left(z_{1}^{2}\right)+v_{3}^{(k)}\left(x_{3}\right)=v_{1}^{(k)}\left(z_{1}^{3}\right)+v_{3}^{(k)}\left(y_{3}\right)
$$

Adding (A-5) and (A-6) and cancelling $v_{1}^{(k)}\left(z_{1}^{2}\right)$ shows that:

$$
v_{1}^{(k)}\left(x_{1}\right)+v_{2}^{(k)}\left(x_{2}\right)+v_{3}^{(k)}\left(x_{3}\right)=v_{1}^{(k)}\left(z_{1}^{3}\right)+v_{2}^{(k)}\left(y_{2}\right)+v_{3}^{(k)}\left(y_{3}\right) .
$$

Iterating the above reasoning easily shows that we must have:

$$
\begin{aligned}
& v_{1}^{(k)}\left(x_{1}\right)+v_{2}^{(k)}\left(x_{2}\right)+\cdots+v_{n-1}^{(k)}\left(x_{n-1}\right)+v_{n}^{(k)}\left(x_{n}\right)= \\
& v_{1}^{(k)}\left(z_{1}^{n}\right)+v_{2}^{(k)}\left(y_{2}\right)+\cdots+v_{n-1}^{(k)}\left(y_{n-1}\right)+v_{n}^{(k)}\left(y_{n}\right) .
\end{aligned}
$$

Because we have $\left(y_{1}, y_{-1}\right) \in C^{h} \cap C^{h+1}$ and $\left(z_{1}^{n}, y_{-1}\right) \in C^{h} \cap C^{h+1}$, generalized thinness implies that $y_{1}$ and $z_{1}^{n}$ cannot be distinguished. In view of the proof 
of Proposition 1, this is easily seen to imply that $v_{1}^{(k)}\left(z_{1}^{n}\right)=v_{1}^{(k)}\left(y_{1}\right)$. Hence, $x, y \in C^{h} \cap C^{h+1}$ imply that

$$
\begin{aligned}
& v_{1}^{(k)}\left(x_{1}\right)+v_{2}^{(k)}\left(x_{2}\right)+\cdots+v_{n-1}^{(k)}\left(x_{n-1}\right)+v_{n}^{(k)}\left(x_{n}\right)= \\
& v_{1}^{(k)}\left(y_{1}\right)+v_{2}^{(k)}\left(y_{2}\right)+\cdots+v_{n-1}^{(k)}\left(y_{n-1}\right)+v_{n}^{(k)}\left(y_{n}\right),
\end{aligned}
$$

so that $\left\langle v_{1}^{(k)}, v_{2}^{(k)}, \ldots, v_{n}^{(k)}\right\rangle$ is not only an additive representation of $\left\langle C_{\leq}^{k}, C_{\geq}^{k}\right\rangle$ but is also an additive representation of $C^{h} \cap C^{h+1}$. It will be convenient to denote by $\lambda_{h}$ the common value given by the sum of the functions $v_{i}^{(k)}$ to all alternatives in $C^{h} \cap C^{h+1}$. Clearly if $h>k$ then $x \in C^{h} \cap C^{h+1}$ implies $x \in \Delta_{\geq}^{k+1}$, so that $\lambda_{h}>0$. Similarly, if $h<k$, we have $\lambda_{h}<0$.

\section{Step 2}

Suppose that $x \in \Delta^{h}$. Let us show that we have $\sum_{i=1}^{n} v_{i}^{(k)}\left(x_{i}\right)<\lambda_{h}$.

Using unrestricted solvability w.r.t. $C^{h} \cap C^{h+1}$, we can find $z_{1} \in X_{1}$ such that $\left(z_{1}, x_{-1}\right) \in C^{h} \cap C^{h+1}$. Using unrestricted solvability w.r.t. $C^{k} \cap C^{k+1}$, we can find $z_{2} \in X_{2}$ such that $\left(x_{1}, z_{2}, x_{-12}\right) \in C^{k} \cap C^{k+1}$. It is impossible that $\left(z_{1}, z_{2}, x_{-12}\right) \in C^{k} \cap C^{k+1}$. Indeed, since $\left(x_{1}, z_{2}, x_{-12}\right) \in C^{k} \cap C^{k+1}$, we would have a clear violation of generalized thinness since $\left(z_{1}, x_{-1}\right) \in C^{h} \cap C^{h+1}$ and $\left(x_{1}, x_{-1}\right) \in$ $\Delta^{h}$. Hence we have either $\left(z_{1}, z_{2}, x_{-12}\right) \in \Delta_{\leq}^{k}$ or $\left(z_{1}, z_{2}, x_{-12}\right) \in \Delta_{\geq}^{k+1}$. Suppose that $\left(z_{1}, z_{2}, x_{-12}\right) \in \Delta_{\leq}^{k}$. We know that $\left(x_{1}, z_{2}, x_{-12}\right) \in C^{k} \cap C^{k+1}$ and $\left(z_{1}, x_{2}, x_{-12}\right) \in$ $C^{h} \cap C^{h+1}$. Using $\Upsilon$-mixed-1-linearity leads to either $\left(z_{1}, z_{2}, x_{-12}\right) \in C_{\geq}^{k+1}$ or $\left(x_{1}, x_{2}, x_{-12}\right) \in C_{\geq}^{h+1}$. This is contradictory since we know that $\left(z_{1}, z_{2}, x_{-12}\right) \in \Delta_{\leq}^{k}$ and $x \in \Delta^{h}$. Hence, we must have $\left(z_{1}, z_{2}, x_{-12}\right) \in \Delta_{\geq}^{k+1}$. Because we know that $\left(x_{1}, z_{2}, x_{-12}\right) \in C^{k} \cap C^{k+1}$, we must have $v_{i}^{(k)}\left(z_{1}\right)>v_{i}^{(k)}\left(x_{1}\right)$. This proves that $\sum_{i=1}^{n} v_{i}^{(k)}\left(x_{i}\right)<\lambda_{h}=v_{i}^{(k)}\left(z_{1}\right)+\sum_{j \neq 1} v_{j}\left(x_{j}\right)$.

\section{Step 3}

Let us show that if $x \in \Delta^{h}$ then $\sum_{i=1}^{n} v_{i}^{(k)}\left(x_{i}\right)>\lambda_{h-1}$.

Using unrestricted solvability w.r.t. $C^{h-1} \cap C^{h}$, we can find $z_{1} \in X_{1}$ such that $\left(z_{1}, x_{-1}\right) \in C^{h-1} \cap C^{h}$. Using unrestricted solvability w.r.t. $C^{k} \cap C^{k+1}$, we can find $z_{2} \in X_{2}$ such that $\left(x_{1}, z_{2}, x_{-12}\right) \in C^{k} \cap C^{k+1}$. It is impossible that $\left(z_{1}, z_{2}, x_{-12}\right) \in C^{k} \cap C^{k+1}$. Indeed, since $\left(x_{1}, z_{2}, x_{-12}\right) \in C^{k} \cap C^{k+1}$, we would have a clear violation of generalized thinness since $\left(z_{1}, x_{-1}\right) \in C^{h-1} \cap C^{h}$ and $\left(x_{1}, x_{-1}\right) \in$ $\Delta^{h}$. Hence we have either $\left(z_{1}, z_{2}, x_{-12}\right) \in \Delta_{\leq}^{k}$ or $\left(z_{1}, z_{2}, x_{-12}\right) \in \Delta_{\geq}^{k+1}$. Suppose that $\left(z_{1}, z_{2}, x_{-12}\right) \in \Delta_{\geq}^{k+1}$. Since $\left(x_{1}, x_{2}, x_{-12}\right) \in \Delta_{\geq}^{h}$, using $\Delta$-mixed-1-linearity leads to either $\left(x_{1}, z_{2}, x_{-12}\right) \in \Delta_{\geq}^{k+1}$ or $\left(z_{1}, x_{2}, x_{-12}\right) \in \Delta_{\geq}^{h}$. This is contradictory since we know that $\left(x_{1}, z_{2}, x_{-12}\right) \in C^{k} \cap C^{k+1}$ and $\left(z_{1}, x_{2}, x_{-12}\right) \in C^{h-1} \cap C^{h}$. Hence, 
we must have $\left(z_{1}, z_{2}, x_{-12}\right) \in \Delta_{<}^{k}$. Since we know that $x \in \Delta^{k}$, this implies that $v_{i}^{(k)}\left(z_{1}\right)<v_{i}^{(k)}\left(x_{1}\right)$. This proves that $\sum_{i=1}^{n} v_{i}^{(k)}\left(x_{i}\right)>\lambda_{h-1}=v_{i}^{(k)}\left(z_{1}\right)+\sum_{j \neq 1} v_{j}\left(x_{j}\right)$.

\section{Step 4}

Since we have supposed that $\left\langle C^{k}\right\rangle_{k \in R}$ is non-degenerate, we know that for all $h \in R$, we have $x \in \Delta^{h}$, for some $x \in X$. Hence the last two steps show that $\left\langle v_{1}^{(k)}, v_{2}^{(k)}, \ldots, v_{n}^{(k)}\right\rangle$ is not only an additive representation of $\left\langle\Delta_{\leq}^{k}, C^{k} \cap C^{k+1}, \Delta_{\geq}^{k+1}\right\rangle$ in model $(A)$ but is also an additive representation of $\left\langle C^{k}\right\rangle_{k \in R}$ in model $\left(A^{*}\right)$.

\section{C.2 Proof of Theorem 3: uniqueness}

It is obvious that if $\left\langle u_{i}\right\rangle_{i \in N}$ is an additive representation of $\left\langle C^{k}\right\rangle_{k \in R}$ using the thresholds $\sigma^{1}<\sigma^{2}<\cdots<\sigma^{r-1}$ then $\left\langle u_{i}+\beta_{i}\right\rangle_{i \in N}$ with $\sum_{i=1}^{n} \beta_{i}=0$ is another additive representation of $\left\langle C^{k}\right\rangle_{k \in R}$ using the same thresholds.

Let $k$ be the index of a twofold covering satisfying the conditions of Part 2 of Theorem 3. It is clear that any additive representation $\left\langle u_{i}\right\rangle_{i \in N}$ of $\left\langle C^{k}\right\rangle_{k \in R}$ is also a representation of $\left\langle C_{\leq}^{k}, C_{>}^{k+1}\right\rangle$ using the threshold $\sigma_{k}$ for $C^{k} \cap C^{k+1}$. We know from Theorem 1 the uniqueness of this representation. We may add a constant $\beta_{i}$ to each $u_{i}$ provided that $\sum_{i=1}^{n} \beta_{i}=0$. We may also multiply each of the $u_{i}$ by the same positive constant $\alpha$. Because we have here fixed the value of each $x \in C^{\ell} \cap C^{\ell+1}$ to the constant $\sigma_{\ell}$, such a dilatation is now incompatible with keeping the same thresholds. Hence, we must take $\alpha=1$.

As above, the uniqueness result given above relies on keeping the thresholds $\sigma^{1}<\sigma^{2}<\cdots<\sigma^{r-1}$ fixed. When they are not, one may choose arbitrarily two of them, via the multiplication of all $u_{i}$ by the same positive constant and the addition of a constant $\beta_{i}$ to each $u_{i}$. The value of the remaining thresholds is then determined by these choices.

\section{References}

Belton, V. and Stewart, T. (2001). Multiple criteria decision analysis: An integrated approach. Kluwer, Dordrecht.

Bouyssou, D. and Marchant, Th. (2007a). An axiomatic approach to noncompensatory sorting methods in MCDM, I: The case of two categories. European Journal of Operational Research, 178(1):217-245.

Bouyssou, D. and Marchant, Th. (2007b). An axiomatic approach to noncompensatory sorting methods in MCDM, II: More than two categories. European Journal of Operational Research, 178(1):246-276. 
Bouyssou, D. and Marchant, Th. (2008a). Additive and decomposable conjoint measurement with ordered categories. Cahier du LAMSADE \# 275, Université Paris Dauphine, 58 pages, available from www.lamsade.dauphine.fr/ bouyssou/.

Bouyssou, D. and Marchant, Th. (2008b). Biorders with frontiers. Working paper, LAMSADE, Université Paris Dauphine, 40 pages, available from www.lamsade.dauphine. fr/ bouyssou/.

Bouyssou, D. and Marchant, Th. (2009). Ordered categories and additive conjoint measurement on connected sets. Journal of Mathematical Psychology, 53(2):92-105.

Bouyssou, D., Marchant, Th., Pirlot, M., Tsoukiàs, A., and Vincke, Ph. (2006). Evaluation and decision models with multiple criteria: Stepping stones for the analyst. Springer, New York.

Chateauneuf, A. and Wakker, P. P. (1993). From local to global additive representation. Journal of Mathematical Economics, 22(6):523-545.

Doignon, J.-P., Ducamp, A., and Falmagne, J.-C. (1984). On realizable biorders and the biorder dimension of a relation. Journal of Mathematical Psychology, 28:73-109.

Fishburn, P. C. (1970). Utility theory for decision-making. Wiley, New York.

Goldstein, W. M. (1991). Decomposable threshold models. Journal of Mathematical Psychology, 35:64-79.

Greco, S., Matarazzo, B., and Słowiński, R. (1999). The use of rough sets and fuzzy sets in MCDM. In: T. Gal, T. Hanne, and T. Stewart (Eds.) Multicriteria decision making, Advances in MCDM models, algorithms, theory and applications, pp. 14.114.59. Kluwer, Dordrecht.

Greco, S., Matarazzo, B., and Słowiński, R. (2002a). Multicriteria classification. In: W. Klogsen and J. Zytkow (Eds.) Handbook of data mining and knowledge discovery, pp. 318-328. Oxford University Press, Oxford.

Greco, S., Matarazzo, B., and Słowiński, R. (2002b). Rough sets methodology for sorting problems in presence of multiple attributes and criteria. European Journal of Operational Research, 138:247-59.

Jacquet-Lagrèze, É. (1995). An application of the UTA discriminant model for the evaluation of R\&D projects. In: P. Pardalos et al. (Eds.) Advances in Multicriteria Analysis, pp. 203-211. Kluwer, Dordrecht.

Keeney, R. L. and Raiffa, H. (1976). Decisions with multiple objectives: Preferences and value tradeoffs. Wiley, New York.

Krantz, D. H. (2009). Personal communication. 8 March 2009.

Krantz, D. H., Luce, R. D., Suppes, P., and Tversky, A. (1971). Foundations of measurement, vol. 1: Additive and polynomial representations. Academic Press, New York. Mousseau, V., Słowiński, R., and Zielniewicz, P. (2000). A user-oriented implementation of the ELECTRE TRI method integrating preference elicitation support. Computers \& Operations Research, 27:757-777.

Nakamura, Y. (2004). Trichotomic preferences for gambles. Journal of Mathematical Psychology, 48(6):385-398.

Roy, B. (1996). Multicriteria methodology for decision aiding. Kluwer, Dordrecht. Original version in French: "Méthodologie multicritère d'aide à la décision", Economica, 
Paris, 1985.

Roy, B. and Bouyssou, D. (1993). Aide multicritère à la décision : méthodes et cas. Economica, Paris.

Słowiński, R., Greco, S., and Matarazzo, B. (2002). Axiomatization of utility, outranking and decision-rule preference models for multiple-criteria classification problems under partial inconsistency with the dominance principle. Control and Cybernetics, 31(4):1005-1035.

Vind, K. (1991). Independent preferences. Journal of Mathematical Economics, 20:119135.

Vind, K. (2003). Independence, Additivity, Uncertainty. Springer-Verlag, Berlin.

von Winterfeldt, D. and Edwards, W. (1986). Decision analysis and behavioral research. Cambridge University Press, Cambridge.

Zopounidis, C. and Doumpos, M. (2000a). Intelligent decision aiding systems based on multiple criteria for financial engineering. Kluwer, Dordrecht.

Zopounidis, C. and Doumpos, M. (2000b). PREFDIS: A multicriteria decision support system for sorting decision problems. Computers \& Operations Research, 27(7-8):779797.

Zopounidis, C. and Doumpos, M. (2002). Multicriteria classification and sorting methods: A literature review. European Journal of Operational Research, 138:229-246. 